\title{
DETECTION AND MODELING VIBRATIONAL BEHAVIOR OF A GAS TURBINE BASED ON DYNAMIC NEURAL NETWORKS APPROACH
}

\author{
BENRAHMOUNE Mohamed ${ }^{1,2}$, HAFAIFA Ahmed ${ }^{1,2}$, \\ GUEMANA Mouloud ${ }^{3}$, CHEN XiaoQi ${ }^{4}$ \\ Applied Automation and Industrial Diagnostics Laboratory, University of Djelfa, Algeria \\ ${ }^{2}$ Gas Turbine Joint Research Team, University of Djelfa, Djelfa, Algeria, \\ e - mails: M.benrahmoune@univ-djelfa.dz,hafaifa.ahmed.dz@ieee.org \\ ${ }^{3}$ Science and Technology Faculty, Médéa University, Algeria, \\ e - mail: guemana.mouloud@univ-medea.dz \\ ${ }^{4}$ Department of Mechanical Engineering, University of Canterbury, Christchurch, New Zealand, \\ e-mail:xiaoqi.chen@canterbury.ac.nz
}

\begin{abstract}
During the gas turbine exploitation the presence of small defects can cause very high vibration amplifications, localized on the components of this rotating machine. For this, a diagnostic process is necessary for decision-making during the monitoring of failures caused by vibration phenomena, which consists in observing the system by comparing its current data with the data coming from a normal operation. These indicators help engineer to determine the symptoms for the failing components of the system. This work deals with problems related to these vibrations, with the aim of developing a system of detection of failures using dynamic neural networks approach. The originality of this contribution is to calculate the various alarms based on this system which used the determined vibration models in order to ensure a reliable and safe operation of the gas compression installation using the examined gas turbine.
\end{abstract}

KEYWORDS: Gas turbine, vibrations monitoring, model based faults detection, dynamic neural networks.

\section{Introduction}

Vibration monitoring is still a hot topic in most industrial sectors, using rotating machines, this domain is designed to provide protective measures against these basic instability phenomena and provides the same level of information for their diagnosis of the thorough dynamic behavior performed on these rotating machines. For this end, this work aims to increase the capacity to monitor the vibratory behavior of a gas turbine under examination. We will focus on the model causes of the axial and radial vibrations of the bearings of this rotating machine, because presence of small defects in these components can lead to very large vibratory amplifications, localized on the whole turbine, with the aim of developing a system of detection of failures using dynamic neural networks, based on the use of actual site data, we will treat the example of the gas compression station at Hassi R'Mel Algeria. This will allow us to better manage the failures caused by vibrations, by detecting defects at an early stage, before they become critical and describe the parameters related to vibration and represents the state of the vibratory motion. In order to find the optimum vibration band which minimizes the vibration thresholds for the diagnosis of defects and to ensure reliable and safe operation in gas compression installations to the economic recovery of the transported gas.

Indeed, in several industrial applications there are methods which have already been developed previously for improving the gas turbine monitoring systems to give the status and 
operating information of a gas turbine from these signals vibrations [1-5]. Nonetheless, these methods have several disadvantages in their starting to integrate the source of vibration into these monitoring systems. However, when a fault occurs on an equipment, the experts go to the site to look for indications that could help them to identify the actual causes of the failure. Specifically, having an idea of the type of failure, they look for the probable causes for the eliminated. To this end, the detection process has the role of predicting the future situation of a process after each detection of change in behavior of the process.

Recently in 2018, Delvecchio S. et al. in [6] proposed a vibro-acoustic state control strategy for internal combustion engines, Yongjia Wu et al. in [7] used the thermoelectric energy modeling for proposed a detection and monitoring system for gas turbines and Zhiyang Guo et al. in [8] analyzed the effects of non-linear instability of a rotor supported by leaf bearings to see their influence on sub-synchronous vibrations. In 2017, several works were realized on the modeling of these rotating machines for the purposes of diagnosis and detection of defects in the objective of avoiding degradation of the performances of the system; product quality, availability and stability. Among these works, Pesaresi L. et al. in [9], proposed a modeling of the nonlinear behavior of a turbine, Nadji Hadroug et al. in [10] linearized the dynamic model of two gas turbine shafts through their input / output data around equilibrium points, Mohammadreza Tahan et al. in [11] carried out a system of monitoring the state of a gas turbine, in two aspects (diagnoses and prognosis), applied to the conditional maintenance of gas turbines.

Although other and different works have been done in this area of diagnosis to evaluate the performance of the industrial system based on performance indicators, using methods to detect and isolate the cause of performance degradation of the system, and allows to predict the causes of these degradations.

In this work, the development of axial and radial vibration models of the turbine bearings is carried out to analyze the vibratory behavior of this machine based on dynamic neural networks, for efficient decision-making during the gas turbine monitoring from the actual operation data of this machine. For reliable analysis and identification of turbine system vibration indicators, to avoid deterioration of their performance, approved by robust testing and validation. This method makes it possible to obtain the information on the states of the system which is useful for its monitoring, while ensuring their performance, which are strongly linked to the functional states during the implementation of this surveillance strategy.

\section{Gas turbine installation}

In the industry, several types of gas turbines are used, for example in gas transportation, chemical processes, electricity generation, the aerospace industry and other applications. Understanding the vibratory behavior of a turbine is an industrial challenge that has continued to increase in importance in these industrial sectors. Uncontrolled dynamic deformation of the structures of the bearings and fins of a turbine can lead to premature aging of these components or to unacceptable noise and vibratory nuisances with non-linearity's present in the turbine system which are of different natures ; Combustion instabilities, such as the air / gas ratio which is responsible for the emission of nitrogen dioxide in large quantities and the instabilities related to the aero elasticity; Floating which begins with the natural frequency of the blade of the rotor with aerodynamic instabilities. These instability can cause deterioration of this machine, so it is essential to keep gas turbine operation outside of the instability zone.

To prevent this phenomenon, gas turbines are equipped with monitoring systems to continuously monitor the mode of operation of these machines [12-18]. Indeed, the solutions proposed so far to prevent this phenomenon have not given encouraging results, because these 
systems always present a risk of passage in vibrational phase. In this work, we propose a method for the diagnosis of the failures of a gas turbine based on artificial neural networks for the improvement of their monitoring.

This will allow us to develop an original method to maintain and monitor gas turbines, in the event of failure or accident to prevent in real time. This approach involves operating the turbine within the acceptable vibration margin, close to the instability zone to ensure the maximum efficiency of the machine, it is a matter of widening the operating area which can reduce the running costs of the turbine. This work is oriented in this way to illustrate and show how in a maintenance policy the vibrations are measured, processed, monitored and used for diagnosis in order to appreciate the mechanical state of the gas turbine. The phenomena of instability and criticism in particular fatigue, wear are amplified by the phenomena of vibration of the parts under stress in the gas turbines [19].

The examined gas turbine in this work is a two-shaft GE 3002 turbine installed in a gas compression station at Hassi R'mel in southern Algeria. This turbine operates at high rotational speeds, it is strongly affected by defects caused by their vibrations, consisting of a 15-stage axial compressor, a combustion chamber arranged $\left(90^{\circ}\right)$ by supplying to the axial high-pressure turbine direction, which comprises a single stage, drives the compressor and its auxiliary equipment with a low-pressure turbine driving the load. The configuration of this installation is shown in Figure 1 and the examined turbine characteristics are given in Table 1.

Table 1: MS 3002 Gas turbine technical characteristics

\begin{tabular}{ll}
\hline Manufacturer: General Electric & Power Turbine : $80-9400 \mathrm{CV}$ \\
\hline Model: MS 3002 & $\begin{array}{l}\text { Fuel consumption rate (100\% HP on 27C): } 2.84 \\
\mathrm{~m}^{3} / \mathrm{s}\end{array}$ \\
\hline
\end{tabular}

Serial No: 244370

Max exhaust temperature: $516.6 \mathrm{C}$

HP Speed and axial compressor: 7100rpm Exhaust gas pressure: 1.009. 3 bar

$\mathrm{N}^{\circ}$ axial compressor stage: $15 \quad$ Starting system: turbo stator

Number of wheels HP wheel (s): $01 \quad$ Direction of rotation: anticlockwise

Speed LP: 6500 rpm Number of shafts: 02 Twin shaft

Measurements of the vibrations carried out on the examined turbine at full speed using the accelerometer shown in Figure 2, the vibrations measured in this machine are primarily related to the quality of the dynamic balancing of the rotating parts, the proximity of a critical speed, to the stiffness of the bearings. 


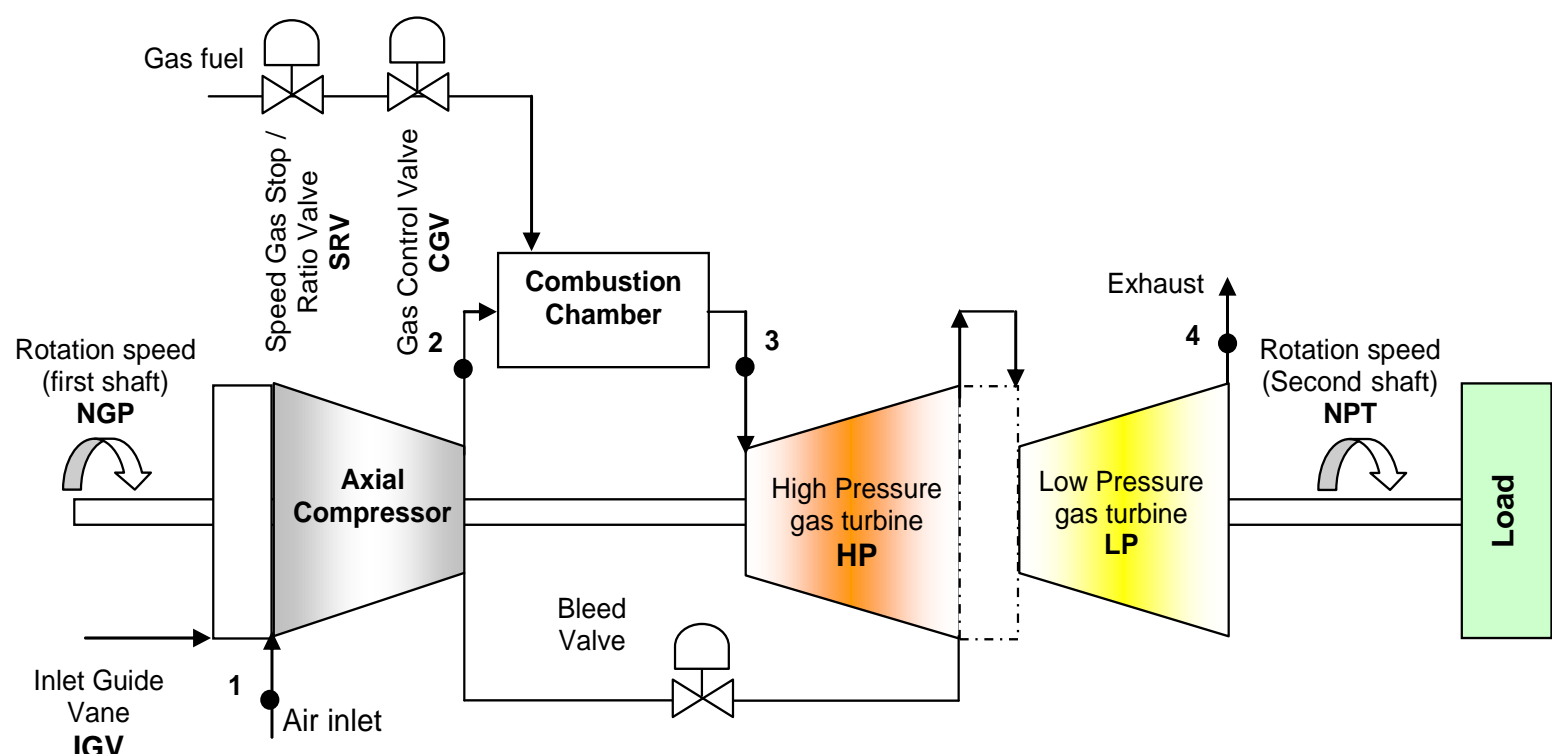

Fig. 1 Two shaft gas turbine

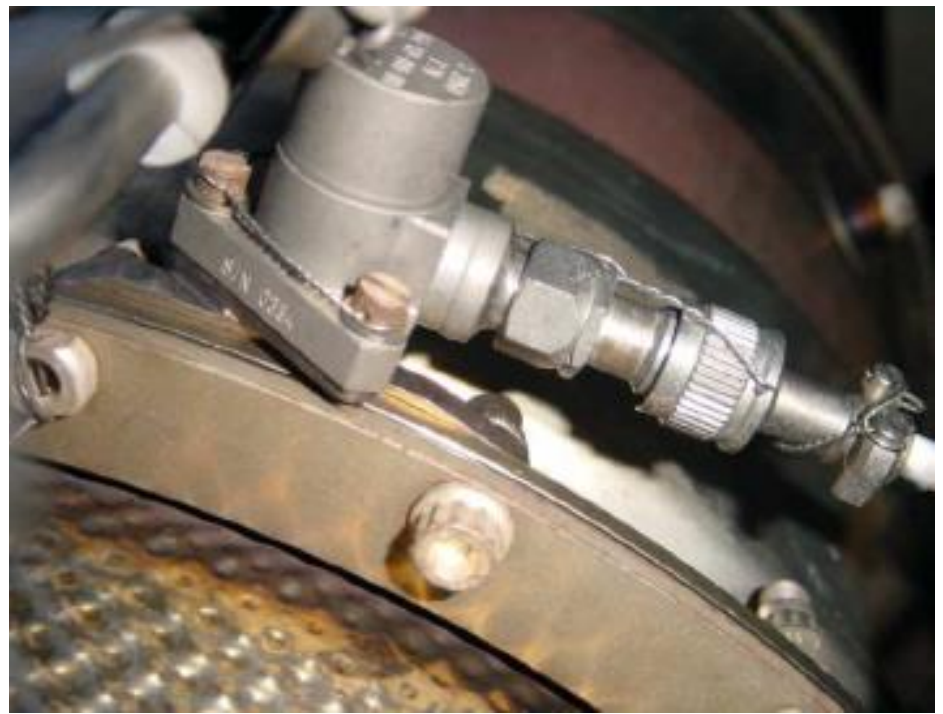

Fig. 2 Accelerometer installed on the tested turbine for vibration measurements

The particularity of rotating machines is the dependence of the parameters of the vibration treatment on the rotational speed. This implies for the system of analysis of the dynamic behavior of a turbine the use of reliable vibration models for the treatment of these vibratory signals, the objective of which is to determine the amplitude and the phase of the vibrations in order to limit the vibrations Vibratory resonance hazards.

\subsection{Vibration of gas turbine components}

The rotating part of the machine is composed of high pressure and low pressure shafts, on which are mounted bladed wheels, where the various bodies of the shaft line are supported by bearings. As mentioned above, the turbine is sufficiently slender to represent it by rotating beams and the shaft can not be perfectly balanced due to the presence of residual unbalances on the shaft line. This is taken into account in the proposed modeling by adding point masses.

In general, and in order to describe the dynamics of a turbine shaft system, the preliminary and necessary step of the modeling consists in defining the parameters and the marks associated with the rotor movement $[11,14]$. Thus, the marks corresponding to a beam in the tri-dimensional space, as shown in Figure 3. 


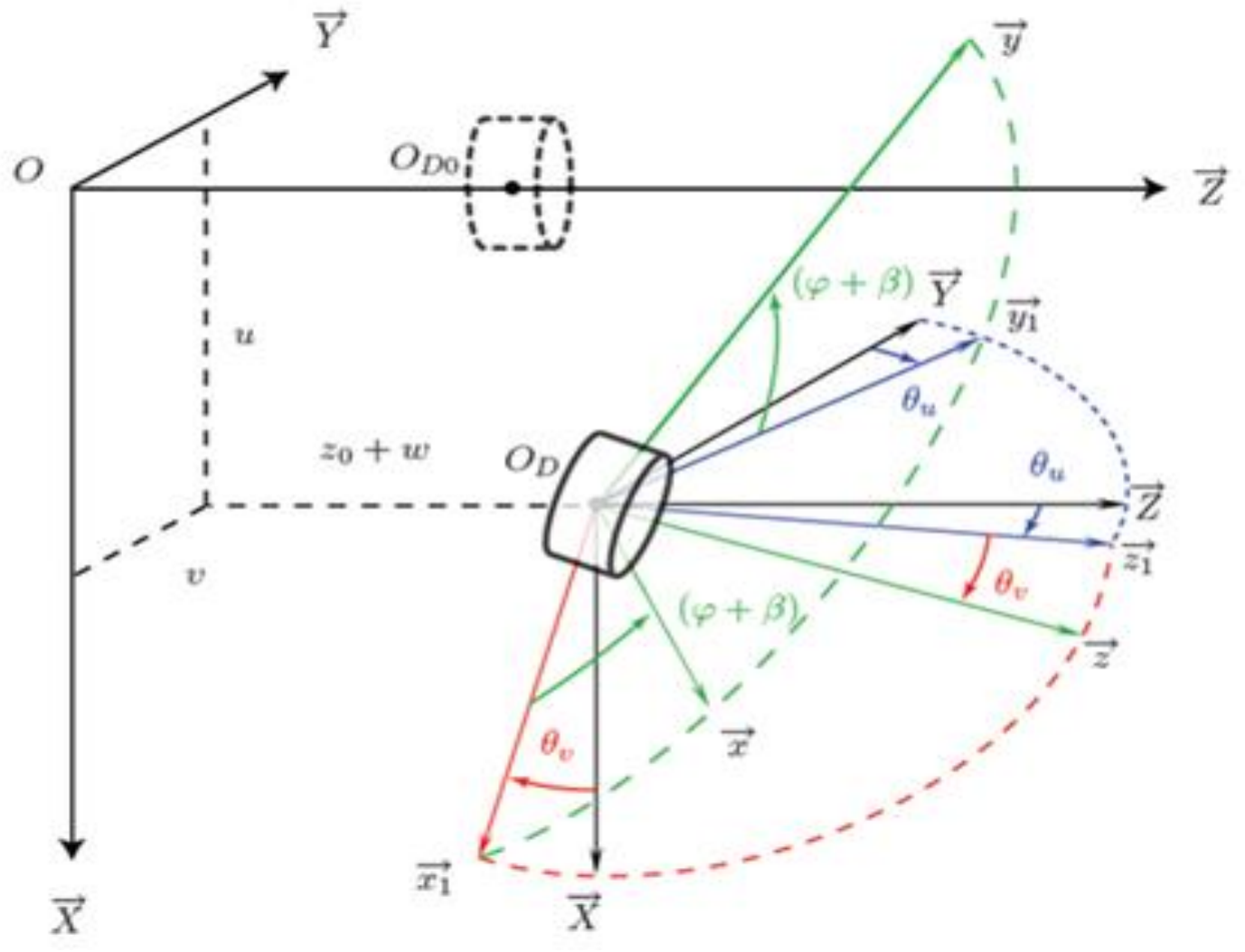

Fig. 3 Notations for a conventionally used disc

The proper rotation of the beam $\theta_{w}$ is composed of the rotation of the rigid body of the shaft $\varphi$ and the angle of torsion $\beta$. By definition, the rotation speed of the solid is written as $[10,14]$ :

$$
\Omega=\dot{\theta}_{u} \vec{X}+\dot{\theta}_{v} \vec{y}_{1}+(\dot{\varphi}+\dot{\beta}) \vec{z}
$$

With the relations of passages between the different plane given by:

$$
\begin{aligned}
\vec{X} & =\cos \theta_{v} \cdot \vec{x}_{1}+\sin \theta_{v} \cdot \vec{z} \\
\vec{x}_{1} & =\cos (\varphi+\beta) \cdot \vec{x}-\sin (\varphi+\beta) \\
\vec{y}_{1} & =\cos (\varphi+\beta) \cdot \vec{x}+\sin (\varphi+\beta)
\end{aligned}
$$

As well as the components of the rotation speed in the plane $R$ are given by:

$$
\vec{\Omega}_{R / R_{g}}^{R}=\left\{\begin{array}{l}
\dot{\theta}_{u} \cos \theta_{v} \cos (\varphi+\beta)+\dot{\theta}_{v} \sin (\varphi+\beta) \\
-\dot{\theta}_{u} \cos \theta_{v} \sin (\varphi+\beta)+\dot{\theta}_{v} \cos (\varphi+\beta) \\
\dot{\varphi}+\dot{\beta}+\dot{\theta}_{u} \sin \theta_{v}
\end{array}\right\}
$$

Wherein $\vec{Z}$ is the neutral axis of the rotor in its initial configuration, $\vec{X}$ and $\vec{Y}$ are the main axes of inertia of the rotor under consideration, $u, v$ and $w$ are respectively the displacements along the axes $\vec{X}, \vec{Y}$ and $\vec{Z}$ of the point of intersection between the cross- 
axial axis of the shaft line, $R_{g}(X, \vec{Y}, \vec{Z})$ is the absolute coordinate system linked to a crosssection of the beam of the initial configuration, $R_{1}\left(\vec{x}_{1}, \overrightarrow{y_{1}}, \overrightarrow{z_{1}}\right)$ is an intermediate rotation plane and $R(\vec{x}, \vec{y}, \vec{z})$ is the reference point linked to the cross-section of the beam of configuration which is deformed in rotation. The transition from the plane $R_{g}$ to the plane $R$ is done by three successive rotations corresponding to the angles of Euler.

For an infinitely rigid circular disc subjected to a variable speed of rotation, the disc being in-deformable, the result is zero deformation energy. Using the notations introduced in Figure 3 , the displacement field associated with the disk is represented in the following form:

$$
\overrightarrow{O O_{D}}=\overrightarrow{O O}_{D O}+\vec{u}=\left\{\begin{array}{l}
0 \\
0 \\
z_{0}
\end{array}\right\}_{R_{g}}+\left\{\begin{array}{l}
u \\
v \\
w
\end{array}\right\}_{R_{g}}=\left\{\begin{array}{l}
u \\
v \\
w+z_{0}
\end{array}\right\}_{R_{G}}
$$

By applying the Huygens formula, the kinetic energy of the disk is obtained by adding the translation energy of the solid to the rotational energy is given by [2]:

$$
2 E_{C / R_{g}}^{D}=M_{D} \vec{V}_{O_{D}}^{R_{G}} \cdot \vec{V}_{O_{D}}^{R_{g}}+\vec{\Omega}_{R / R_{g}}^{R} \cdot\left(\left[J_{/ O_{D}} \cdot \vec{\Omega}_{R / R_{g}}^{R}\right]\right)
$$

Where $M_{D}$ is the mass of the disk, $\vec{V}_{O_{D}}^{R_{g}}=\frac{d}{d t} \overrightarrow{O O}_{D_{I R_{g}}}$ is the instantaneous velocity vector with $O$ fixed in $R_{g}$ and $\left[J_{/ O_{D}}\right]$ is the inertia operator of the disk.

The principal axes of the disc being $x, y$ and $z$, taking into account the symmetries, the inertia operator is written by:

$$
\left[j / O_{D}\right]=\left[\begin{array}{ccc}
I_{D x} & 0 & 0 \\
0 & I_{D x} & 0 \\
0 & 0 & I_{D x}
\end{array}\right]
$$

Therefore, the exact expression of the kinetic energy will be written by:

$$
\begin{aligned}
& 2 E_{c}^{D}=M_{D}\left[\dot{u}^{2}+\dot{v}^{2}+\dot{w}^{2}\right]+I_{D x}\left[\dot{\theta}_{u}^{2} \cos ^{2} \theta_{v}+\dot{\theta}_{v}^{2}\right]+I_{D z}\left[\dot{\theta}_{u}^{2} \sin ^{2} \theta_{v}+(\dot{\varphi}+\dot{\beta})^{2}\right. \\
& \left.+2(\dot{\varphi}+\dot{\beta}) \dot{\theta}_{u} \sin \theta_{v}\right]
\end{aligned}
$$

By placing ourselves within the framework of small perturbations, this quantity can be approached by a limited development considering all the angular quantities, $\varphi$ except their derivatives, small in front of the unit. It is classical to work with equations of motion linearity's, obtained from energies developed in system order 2.

In the practical case, such an approximation would result in the elimination of terms resulting from the gyroscopic effect $\dot{\varphi} \dot{\theta}_{u} \theta_{v}$, as well as the weak coupling between the different vibrations of the turbine $\dot{\beta} \dot{\theta}_{u} \theta_{v}$. This is why we develop this energy by keeping the terms of system order 3 , then we obtain:

$$
2 E_{C}^{D}=M_{D}\left[\dot{u}^{2}+\dot{v}^{2}+\dot{w}^{2}\right]+I_{D x}\left[\dot{\theta}_{u}^{2}+\dot{\theta}_{v}^{2}\right]+I_{D z}\left[(\dot{\varphi}+\dot{\beta})^{2}+2(\dot{\varphi}+\dot{\beta}) \dot{\theta}_{u} \theta_{v}\right]
$$

In equation (8), the strong coupling between the angular position and the torsional vibration can also be noticed with the total rotation term $(\dot{\varphi}+\dot{\beta})$. 
For the shaft of a gas turbine is modeled by a rotating beam, the general formulation of the kinetic energy of the shaft is obtained by extending the case of the disk [10,11, 14, 20-21]. In this case, the energy of a beam slice, of infinitesimal length $d z$, is that of a disc of the same size. Thus, by integrating this formula along the length of the tree, it comes:

$$
E_{C}=\frac{\rho}{2} \int_{0}^{l}\left[S\left(\dot{u}^{2}+\dot{v}^{2}+\dot{w}^{2}\right)+I_{x}\left(\dot{\theta}_{u}^{2}+\dot{\theta}_{v}^{2}\right)+I_{p}\left((\dot{\varphi}+\dot{\beta})+2(\dot{\varphi}+\dot{\beta}) \dot{\theta}_{u} \theta_{v}\right)\right] d z
$$

The deformation energy of a rotating beam is equal to [8]:

$$
2 E_{d}=\int_{0}^{l}\left[E S w_{, z}^{2}+E I_{x}\left(\theta_{u, z}^{2}+\theta_{v, z}^{2}\right)+G I_{p} \beta_{, z}^{2}+k G S\left(\left(u_{, z}-\theta_{v}\right)^{2}+\left(u_{, z}+\theta_{u}\right)^{2}\right)\right] d z
$$

Where $k$ is the correction factor for shear stiffness.

\subsection{Unbalance phenomenon}

It is chosen to represent the loss of a fin or a residual unbalance resulting from imperfect balancing of the rotor by introducing an unbalance in the form of a point mass $m b$ situated at a distance $r b$ from the axis of rotation. Considering the notations introduced in Figure 4, the displacement field associated with the unbalance is determined by the following relation:

$$
\overrightarrow{O B}_{R_{g}}=\overrightarrow{O C}+\overrightarrow{C B}=\left\{\begin{array}{l}
u+r_{b} \cos (\varphi+\beta) \\
v+r_{b} \sin (\varphi+\beta) \\
w+z_{C}
\end{array}\right\}
$$

Thus the velocity of the unbalance is given by:

$$
\vec{V}_{B / R_{g}}=\left\{\begin{array}{l}
\dot{u}-r_{b}(\dot{\varphi}+\dot{\beta}) \sin (\varphi+\beta) \\
\dot{v}+r_{b}(\dot{\varphi}+\dot{\beta}) \cos (\varphi+\beta) \\
\dot{w}
\end{array}\right\}_{R_{g}}
$$

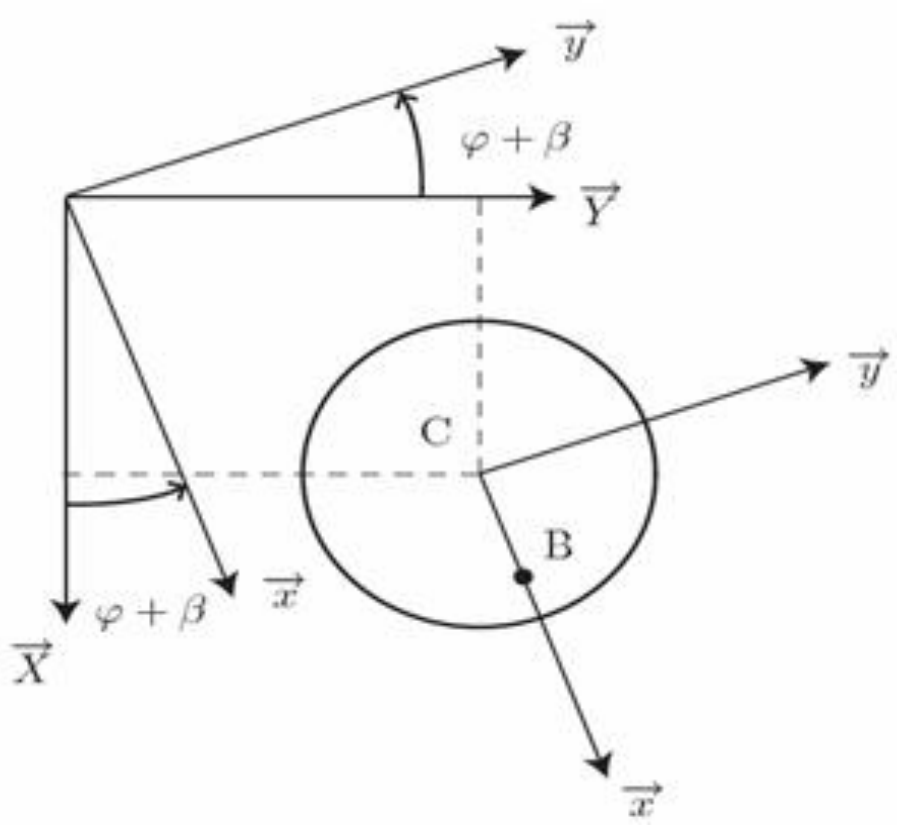

Fig. 4 Unbalance phenomenon notation 
The unbalance being considered as a point mass, its internal energy is zero, the expression of the kinetic energy of the unbalance is given by the following relation:

$$
2 E_{C}^{B}=m_{b}\left\|\vec{V}_{B / R_{g}}\right\|^{2}
$$

Is:

$2 E_{C}^{B}=m_{b}\left[\dot{u}^{2}+\dot{v}^{2}+\dot{w}^{2}+r_{b}^{2}(\dot{\varphi}+\dot{\beta})^{2}+2 r_{b}(\dot{\varphi}+\dot{\beta})(\dot{v} \cos (\varphi+\beta)-\dot{u} \sin (\varphi+\beta))\right]$

Equation (14) shows that the unbalance excites the rotor in torsion and reveals a strong coupling between the torsion angle and the angular position of the shaft. The angle of torsion $\beta$ could be neglected before the angular position $\varphi$ either $\varphi+\beta \cong \varphi$ in this equation (14).

The vibratory behavior of rotating machines is extremely varied, each machine is the seat of dynamic excitations extremely diverse and specific to its operation. The fixed and movable members are vibrating structures, where all these components respond according to its modal characteristics and its damping capacities. However, each vibratory regime generates dynamic stresses that stress the parts in fatigue, it is impossible that a machine, whose design becomes more and more energetic, does not vibrate, but all the know-how of the constructor endeavors to ensure that the fatigue strength capacities can admit these alternating stresses. Subsequent to this work, a vibrational behavior modeling based on neural networks dynamics will be developed to place the influence of the speeds of a gas turbine considering the high and low speed of the rotor of this turbine.

\section{$3 \quad$ Vibrations modeling using dynamic neural networks}

The aim of this work is the use of neural networks for modeling the vibratory behavior of a gas turbine as a predictor of the axial and radial vibration models of the turbine shaft being examined. The vibration monitoring system, shown in Figure 5, communicates state and vibration data to the turbine control adaptation system via a communication adapter module and with Location monitoring modules and identification also called detection module. Also, proximity detection input modules, depending on the vibration data they transmit, such as the radial or axial inputs of the vibration sensors, are used in the vibration defects monitoring system of the examined gas turbine.

In this Figure 5, the identification model is based on dynamic neural networks (NARX), used to detect malfunctions of the machine based on the architecture of the multi-layered perceptron, with feedback to the input. In order to model the turbine vibration behavior, as a predictor of the axial and radial vibration models of the examined gas turbine shaft.

The neural network is designed to perform the monitoring tasks defined by input sequences and sequences of desired values corresponding to the output neurons (reference model) [2225]. The NARX dynamic neural network structure proposed in this work is shown in Figure 6. 


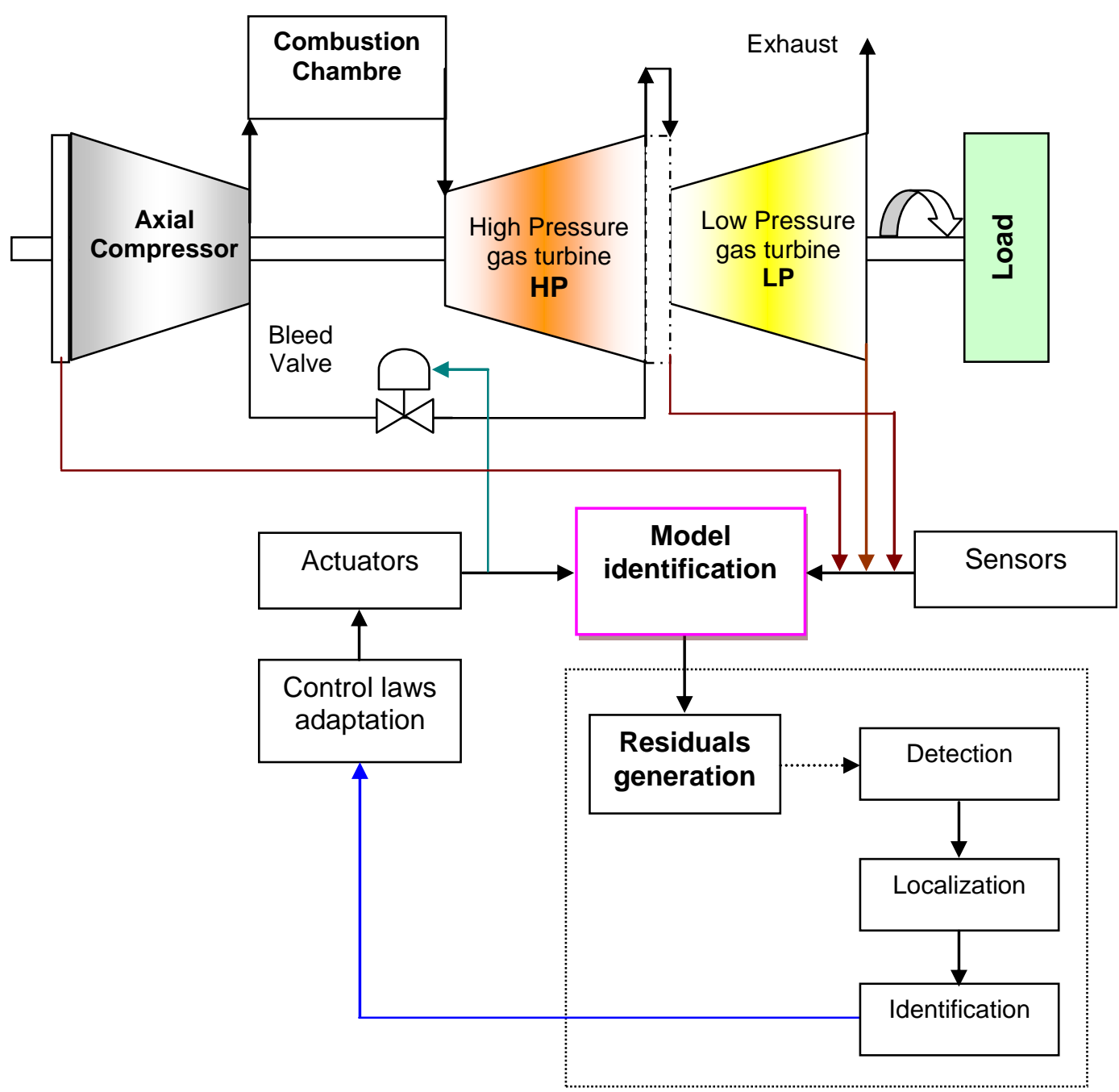

Fig. 5 Fault diagnosis caused by vibrations

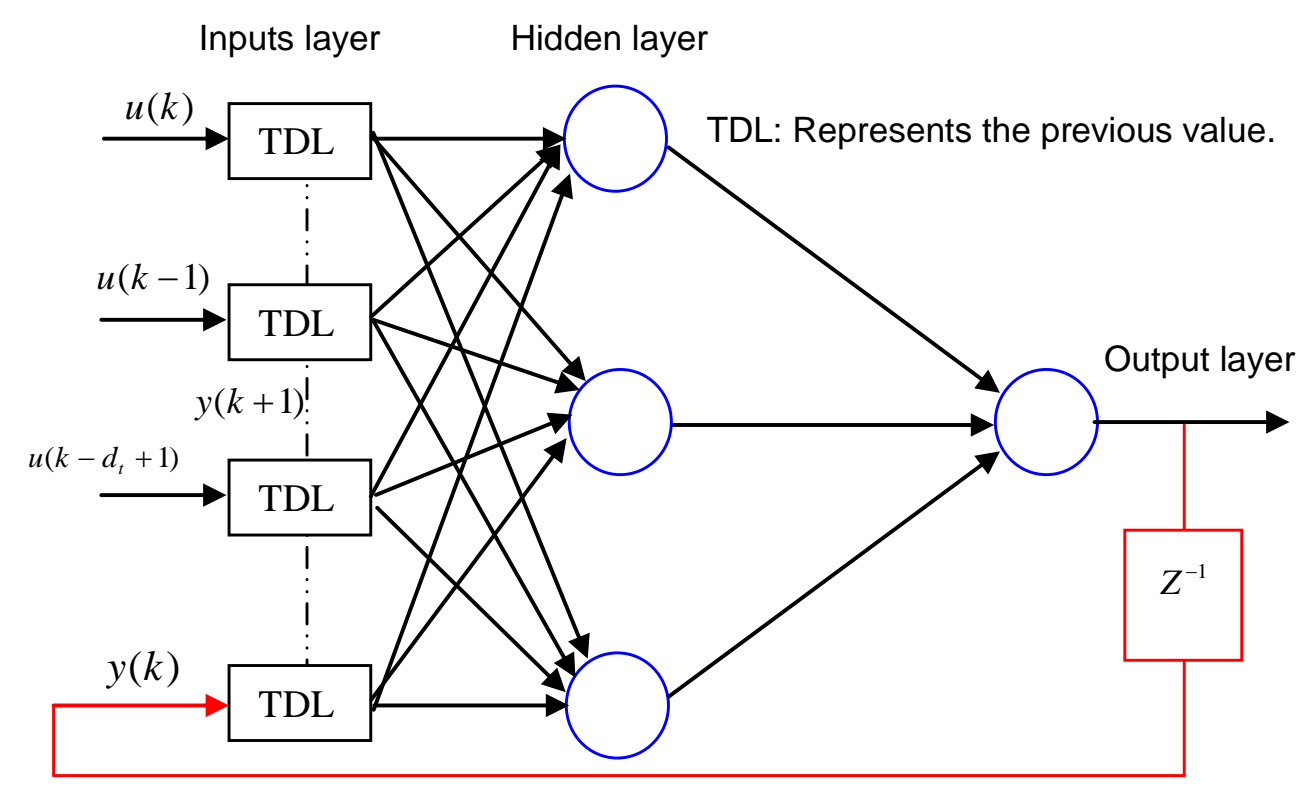

Fig. 6 Dynamic neural networks structure 
The characteristic equation of dynamic neural networks NARX is given by [22, 26-27]:

$$
y(k+1)=f\left[\begin{array}{l}
y(k), y(k-1), \ldots, y\left(k-d_{y}+1\right) \\
u(k), u(k-1), u(k-2) \ldots ., u\left(k-d_{u}+1\right)
\end{array}\right]
$$

With $u(k)$ and $y(k)$ are respectively the input and the output of the networks at the instant $k$, the input and the output of the system at the time instant $n$, with $d_{u} \geq 1$ and $d_{y} \geq 1$ are the input memory and output memory orders with $d_{u} \leq d_{y}$.

The nonlinear activation function $f$ is approximated by a multilayer perception (PMC), the multi-layer neural network is most commonly used for modeling and control with a sigmoid and linear function respectively in the hidden layer and the output layer defined by the following equations:

$$
\begin{aligned}
& u_{j}=f_{1}\left(\sum_{i=1} w_{i j}^{1} x_{i}+w_{k i}^{1} x_{k}+b_{j}^{1}\right) \\
& y_{k}=f_{2}\left(\sum_{i=2}^{N} u_{1}+b_{k}^{2}\right)
\end{aligned}
$$

Where $w_{k j}$ is the synaptic weight between the hidden layer and the output layer, $w_{j i}$ is the synaptic weight between the input layer and the hidden layer, $w_{k i}$ the synaptic weight between the output layer and the input layer ), $x_{i}$ is the connection between the input layer and the hidden layer, $x_{j}$ the connection between the hidden layer and the output layer, $x_{k}$ the connection between the output layer and the input layer, $b_{j}^{1}$ is the bias of the neuron $j, f_{1}$ is the activation of the hidden layer, $f_{2}$ is the activation function of the output layer, $k$ is the index of the output layer, $j$ is the hidden cache layer index, $i$ is the input layer index.

The retro-propagation algorithm is used in this work to minimize the objective function $E$, using the gradient method. This training begins with a random choice of the initial vectors of the weight. The object of the back-propagation algorithm of the error is to adapt the synaptic weights $w$ to minimize the cost function, using the following algorithm:

\section{- Beginning}

$$
\begin{aligned}
& E(W)=\sum_{p=1}^{T} E_{p}(W) \\
& E_{p}(W)=\frac{1}{2} \sum_{i=1}^{m}\left[y_{i}^{d}(k)-y_{i}(k)\right]^{2}
\end{aligned}
$$

- Adaptation of weights by the gradient method using :

$$
\begin{aligned}
& E(W)=\sum_{p=1}^{T} E_{p}(W) \\
& \Delta W_{i j}^{l}=l r \cdot \frac{d E(W)}{d W_{i j}^{l}(k)}
\end{aligned}
$$


$k$ is iteration number and $l r$ is the learning step.

- Derivative of the cost function $E$ by weight ratio $W_{i j}$ is performed by:

$$
\frac{d E(W)}{d W_{i j}^{l}}=\sum_{p=1}^{T} \frac{d E(W)}{d W_{i j}^{l}(k)}
$$

- Derived from the cost function for the hidden layer

$$
\begin{aligned}
& \frac{d E_{p}(k)}{d W_{i j}^{l}}=\sum_{p=1}^{N_{l+1}} \frac{d E_{p}(W)}{d E_{q}^{l+1}(k)} \cdot \frac{d E_{q}^{l+1}(k)}{d E_{i}^{l}(k)} \\
& \frac{d E_{p}(k)}{d W_{i j}^{l}}=f^{l^{\prime}}\left(P_{i}^{l}(k)\right) \cdot U_{j}^{l-1}(k) \\
& \frac{d E_{p}(W)}{d W_{i j}^{l}}=\frac{d E_{p}(W)}{d U_{j}^{l}(k)} \cdot f^{l^{\prime}}\left(P(k) \cdot U_{j}^{l-1}(k)\right)
\end{aligned}
$$

- Derived from the cost function for the output layer

$$
\frac{d E_{p}(k)}{d U_{j}^{l}(k)}=\left(y_{i}^{d}(k)-y_{i}(k)\right)
$$

- End

The data are generated for several robustness tests of the proposed modeling approach; In the absence of noise, in the presence of additive output noise, in the presence of state noise and in the presence of output noise and state noise. For each case, the performances of the NARX structures, in particular the AIC criterion in training and the validation error, are compared in order to check the adequacy between the structure of the nonlinear model and the system to be identified.

The performances of the learning and validation models are evaluated by the square root of the Root Mean Square Error (RMSE) given by [27-28]:

$$
R M S=\sqrt{\frac{1}{N} \sum_{k=1}^{N}(y(k)-\hat{y}(k))^{2}}
$$

where $N$ is the number of observations and $\hat{y}($.$) is the output of the model.$

\section{Application and investigation results}

Gas turbines are widely used in the gas transport sector and in other industrial fields. Nevertheless, these machines are subjected to a very important instability problem caused by the presence of vibration, to characterize and identify the defects from the vibratory signals measured on these machines, it is necessary to model these vibratory behaviors. This makes it possible to elaborate the key steps in the diagnosis of the defects of this type of rotating machine. Generally, these vibratory signals of the defects of rotating machines are generally non-stationary, affecting the rotor of the machine, in the case of a rotor constituted by a flexible shaft and a disk whose center of mass is unbalanced, as is shown in Figure 7, during rotation at a rotational speed $w$. 


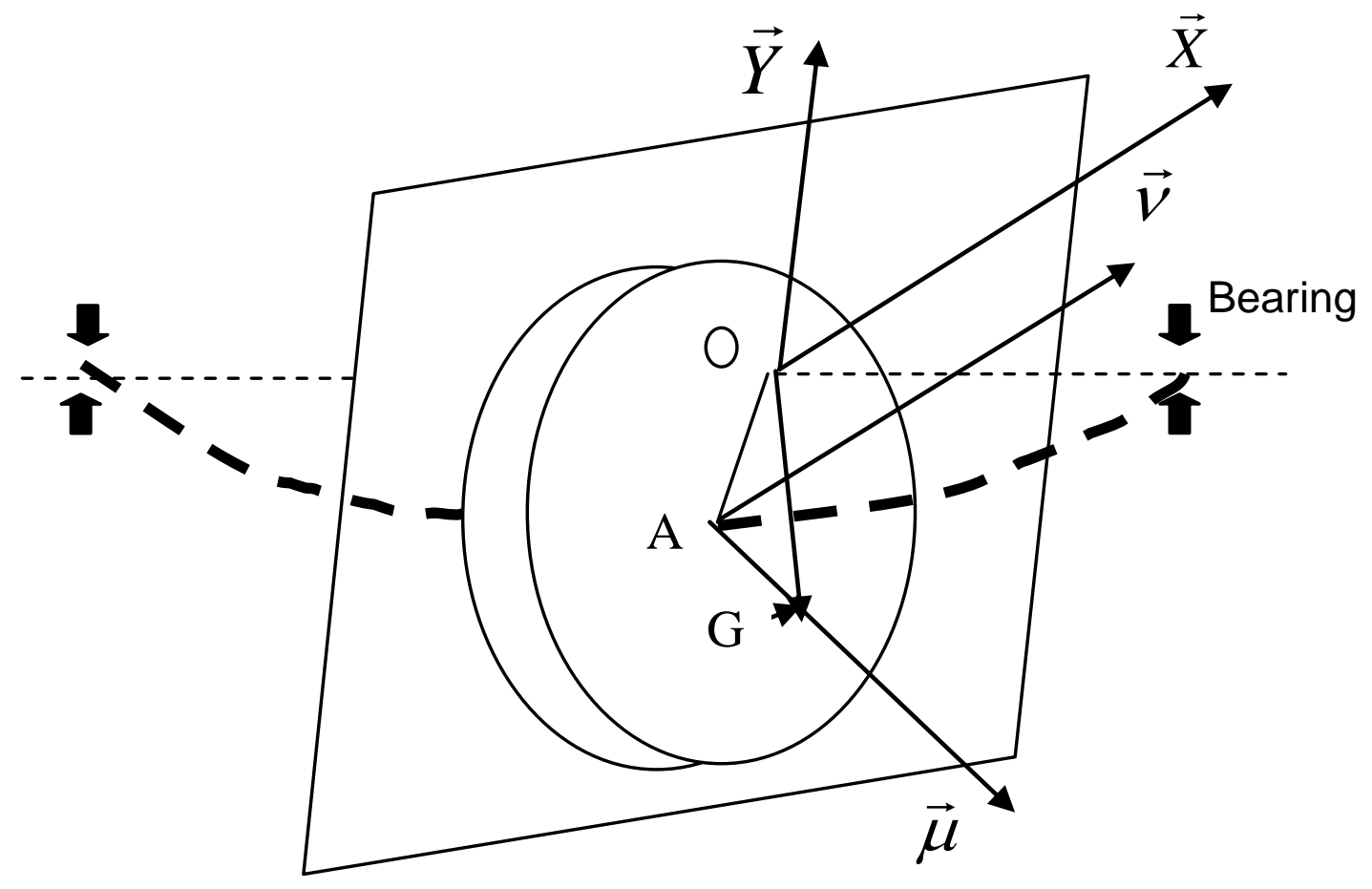

Fig. 7 Movement of a rotating rotor

The forces applied to the system are the stiffness forces, the damping forces and the inertial forces which exert at the center of gravity given by:

$$
\vec{F}=m \cdot \frac{d^{2} \overrightarrow{O G}}{d t^{2}} \text { with } \overrightarrow{O G}=\left\{\begin{array}{l}
X=x+e \cos (\omega t) \\
Y=y+e \cos (\omega t)
\end{array}\right.
$$

By derivation we find:

$$
\overrightarrow{F_{i}}=\left\{\begin{array}{l}
m x^{\prime \prime}-m e \omega^{2} \cos (\omega t) \\
m y^{\prime \prime}-m e \omega^{2} \sin (\omega t)
\end{array} \text { with } \frac{d^{2} \overrightarrow{O G}}{d t^{2}}=\left\{\begin{array}{l}
X^{\prime \prime}=x^{\prime \prime}-e \omega^{2} \cos (\omega t) \\
Y^{\prime \prime}=y^{\prime \prime}-e \omega^{2} \sin (\omega t)
\end{array}\right.\right.
$$

The stiffness coefficient applied to point A (the displacement of point A relative to point $O$ ), the stiffness coefficient of this system depends on (Young's modulus, shaft length, inertia of the shaft) for the conservative case, given by [29]:

$$
\vec{F}=-K \overrightarrow{O A} \text { with } \overrightarrow{O A}=\left\{\begin{array}{l}
x \\
y
\end{array} \text { and } \vec{F}=\left\{\begin{array}{l}
-k x \\
-k y
\end{array}\right.\right.
$$

The damping forces are presented in two external and internal forms, where the external damping has a significant influence on the speed of rotation of the turbine shaft:

$$
\overrightarrow{F_{c}}=-c \cdot \frac{d \overrightarrow{O A}}{d t} \text { with } \frac{d \overrightarrow{O A}}{d t}=\left\{\begin{array}{l}
x^{\prime} \\
y^{\prime}
\end{array} \text { and } \overrightarrow{F_{C}}=\left\{\begin{array}{l}
-c x^{\prime} \\
-c y^{\prime}
\end{array}\right.\right.
$$

Hence $\mathrm{O}$ is the center of rotation, $\mathrm{A}$ is the center of geometry of the disk, $\mathrm{G}$ is the center of gravitation, $(x, y)$ is the fixed reference and $(\vec{\mu}, \vec{v})$ is the mobile reference.

The rotation of a rotor of a rotating machine generates accelerations which are applied to the masses and cause inertia forces. When the axis of rotation does not contain the mass barycentre, its centrifugal acceleration causes an imbalance force with periodic movements in 
the radial direction whose frequency is equal to the speed of rotation $w$. To identify these vibration problems that are difficult to model by analytical methods, the use of techniques derived from the field of artificial intelligence appears as a main alternative for these vibration problems.

In this section, vibration behavior modeling of a gas turbine is developed based on dynamic neural networks, to identify the nonlinear system dynamics of instability phenomena from experimental data to approximate the variables of this nonlinear system By integrating the concept of artificial neural networks, to model the dynamics of this rotating machine. Using the data collected on the sensors of the five bearings (1-5 xy) of the gas turbine tested, as shown in Figure 8.

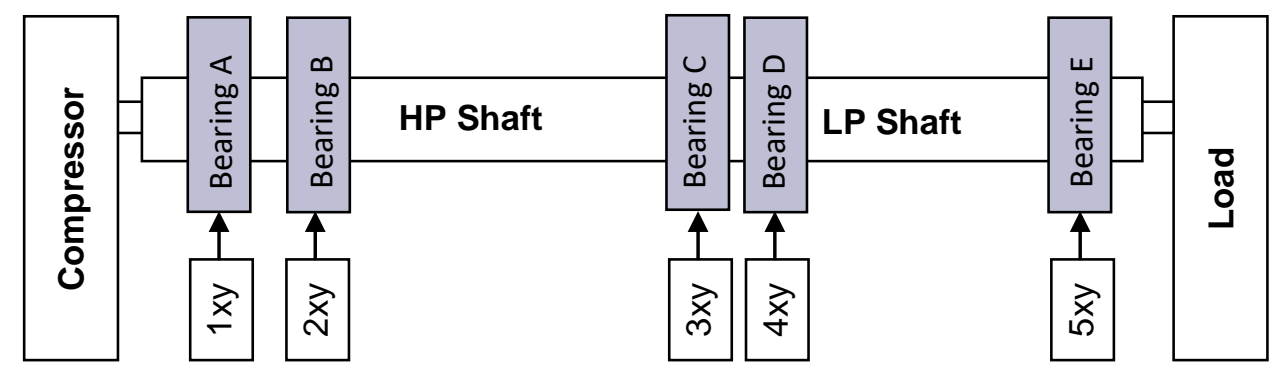

Fig. 8 Sensor bearing of the examined gas turbine

The proposed architecture of the NARX neural network algorithm for the vibration monitoring task of the examined turbine is shown in Figure 9, this configuration uses a predictor learning associated with a multi-input / multi-output process. Since the architecture of the network is fixed, the purpose of learning is to estimate the coefficients to best fulfill the task for which the network is intended.

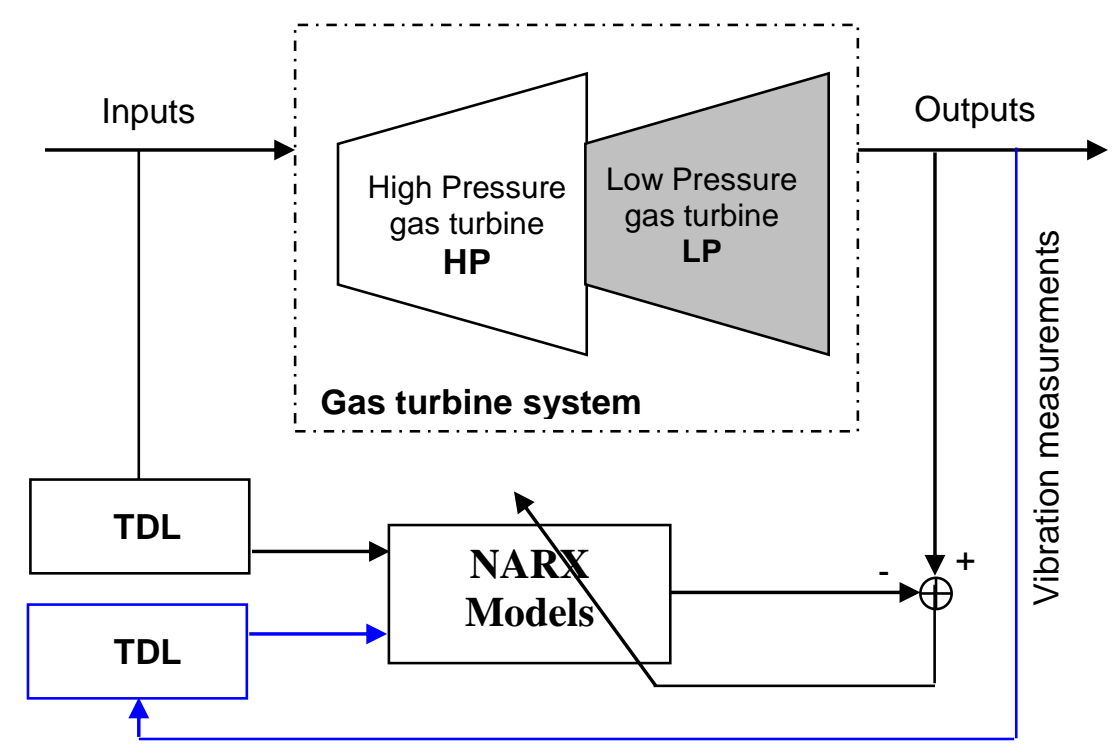

Fig. 9 Turbine vibration monitoring architecture

The following two Figures 10 and 11 show the evolution of actual outputs of the speed of rotation of the high-pressure turbine HP and the speed of the low-pressure turbine LP used subsequently in the axial and radial vibration modeling of the five bearings of this turbine. 


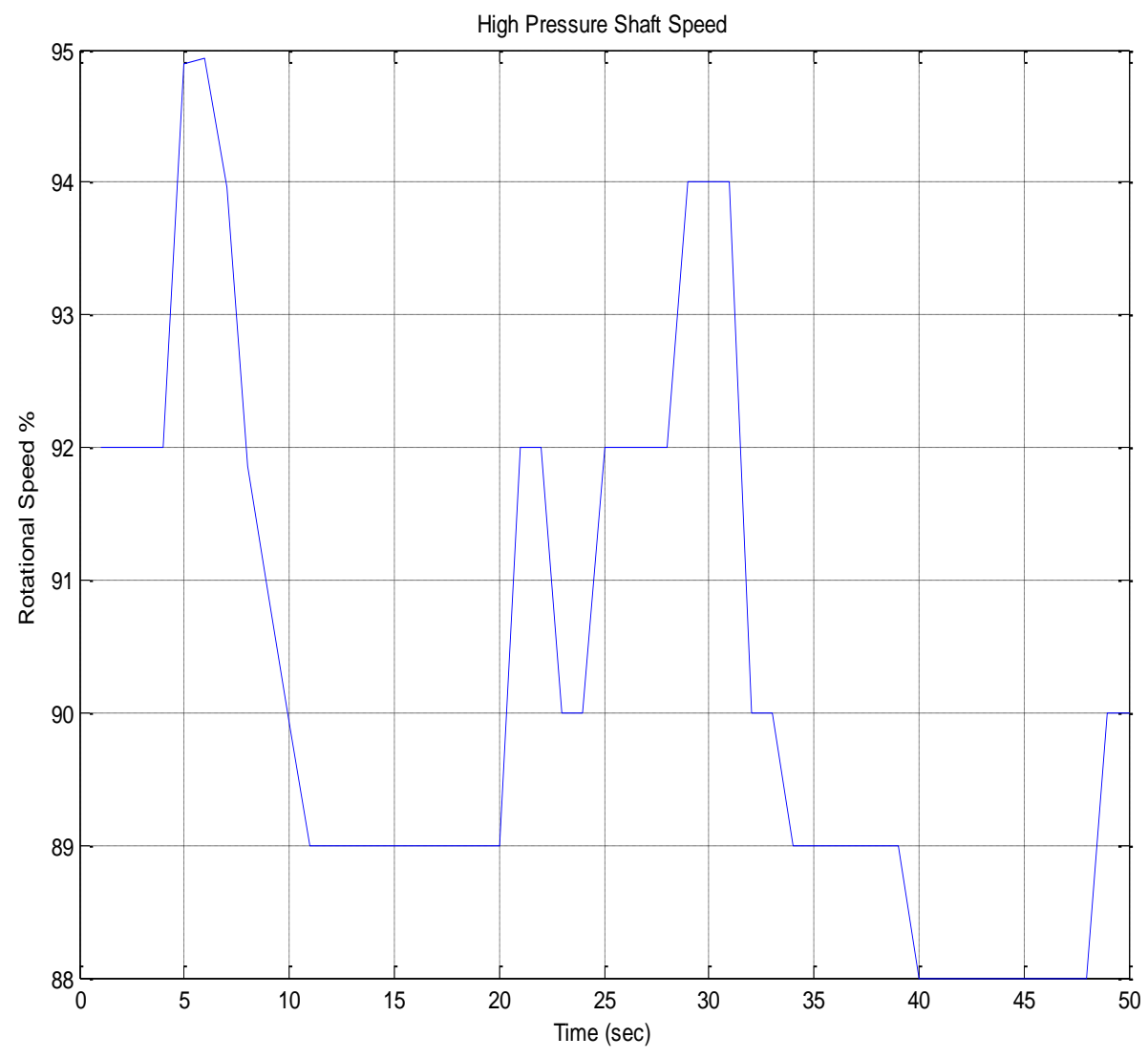

Fig. 10 Vitesse de turbine haute pression

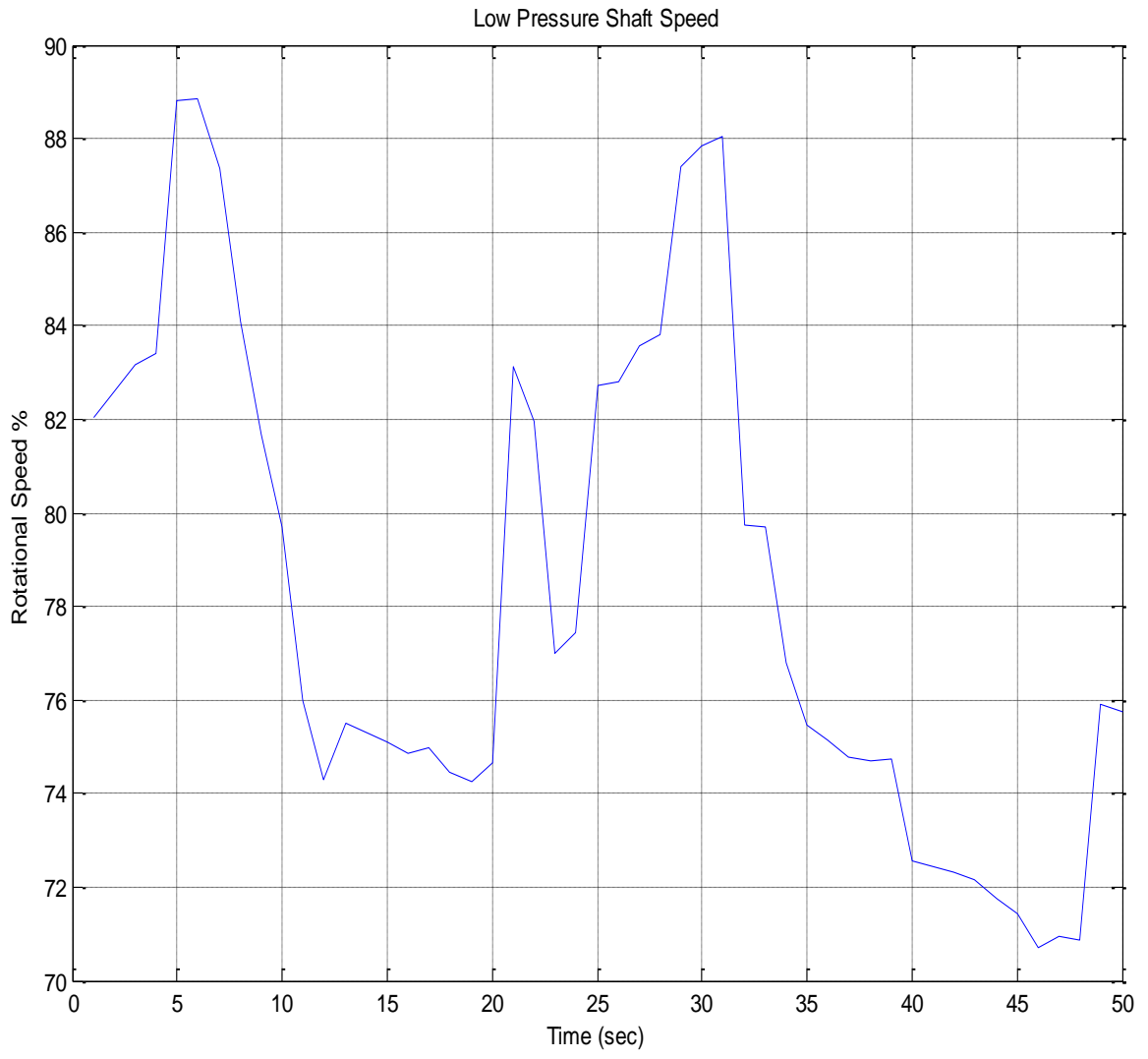

Fig. 11 Vitesse de turbine haute pression 
The application data are restricted by the minimum and maximum of each bearing vibration variable of the gas turbine under consideration (axial or radial vibration) and corresponds to the range of validity of the neural obtained model for each stage. For each stage, an interval was defined according to the observed measurement values.

The obtained results fro vibration modeling of the five bearings show in Figures 12 to Figure 21. In Figure 12, the axial vibratory signals of bearing A of the high-pressure turbine exceed the tolerance range of $25 \mathrm{um}$, this allows generated operating alarms in degraded mode of the machine and Figure 13, shows the obtained neural model response of stage A, this model perfectly reproduces the entire operating mode of the turbine by efficient learning and this is given by the modeling error.

Figure 14 shows the axial and radial vibratory signals of bearing B of the high-pressure turbine with a peak of $24.5 \mu \mathrm{m}$ of axial vibration, with the used network structure, a very good modeling error can be seen in Figure 15, which confirms the effectiveness of the model for the axial and radial vibrations of bearing $\mathrm{B}$.

Figure 16 shows the axial and radial vibratory signals of bearing $\mathrm{C}$ of the high pressure turbine with a peak of $17.5 \mu \mathrm{m}$ of axial vibration and $15.6 \mu \mathrm{m}$ of radial vibration, these results corresponding to the different operating conditions of degraded mode used in the bases learning and testing, in order to demonstrate the good behavior of the neural model under all conditions and to validate its possibilities for generating the vibration indicators, Figure 17 shows the obtained neural model response of stage $\mathrm{C}$ with an modeling error of zero, which confirms the efficiency of the model obtained for the axial and radial vibrations of this bearing $\mathrm{C}$. 


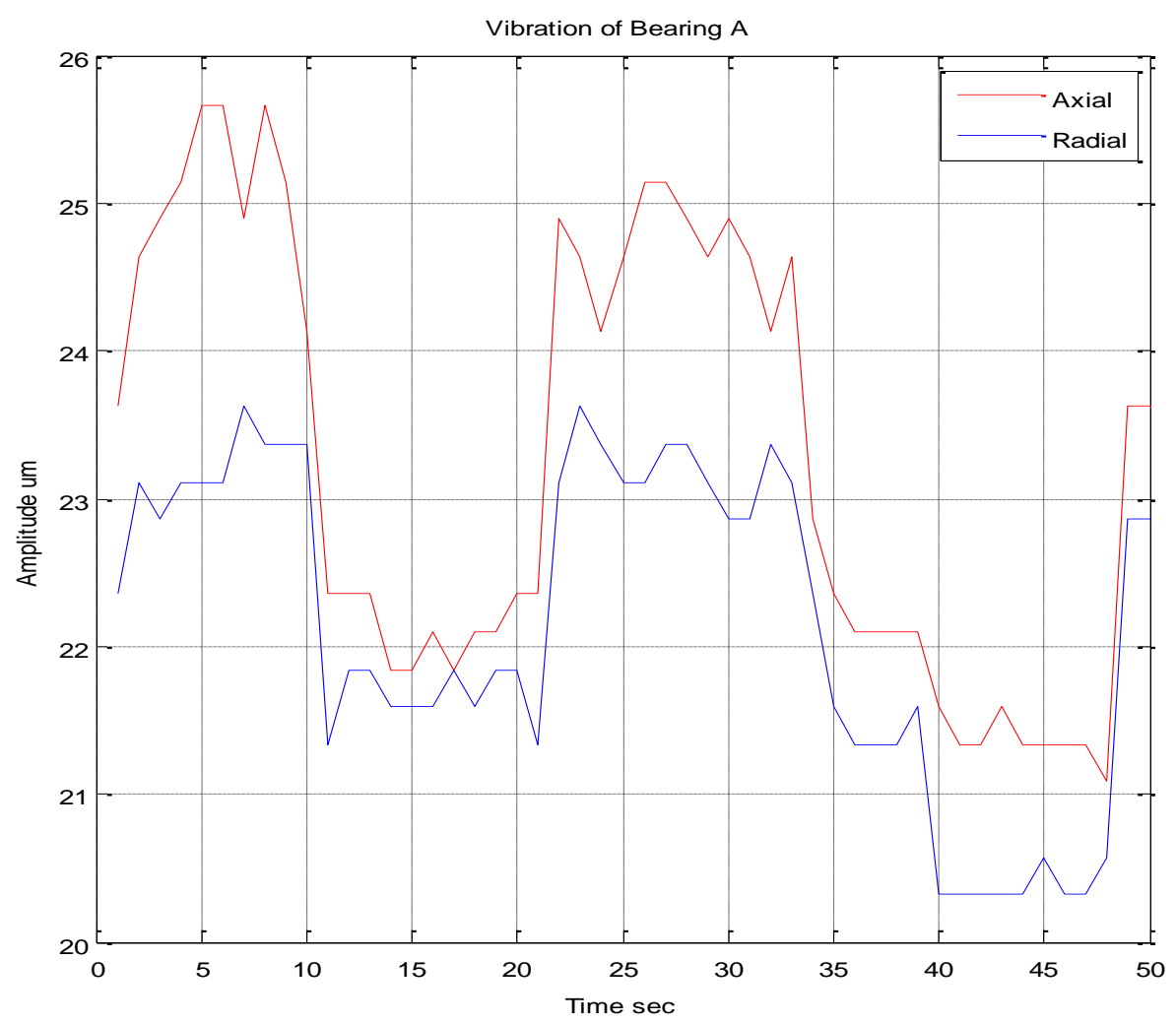

Fig. 12 Variation of axial and radial response of bearing A

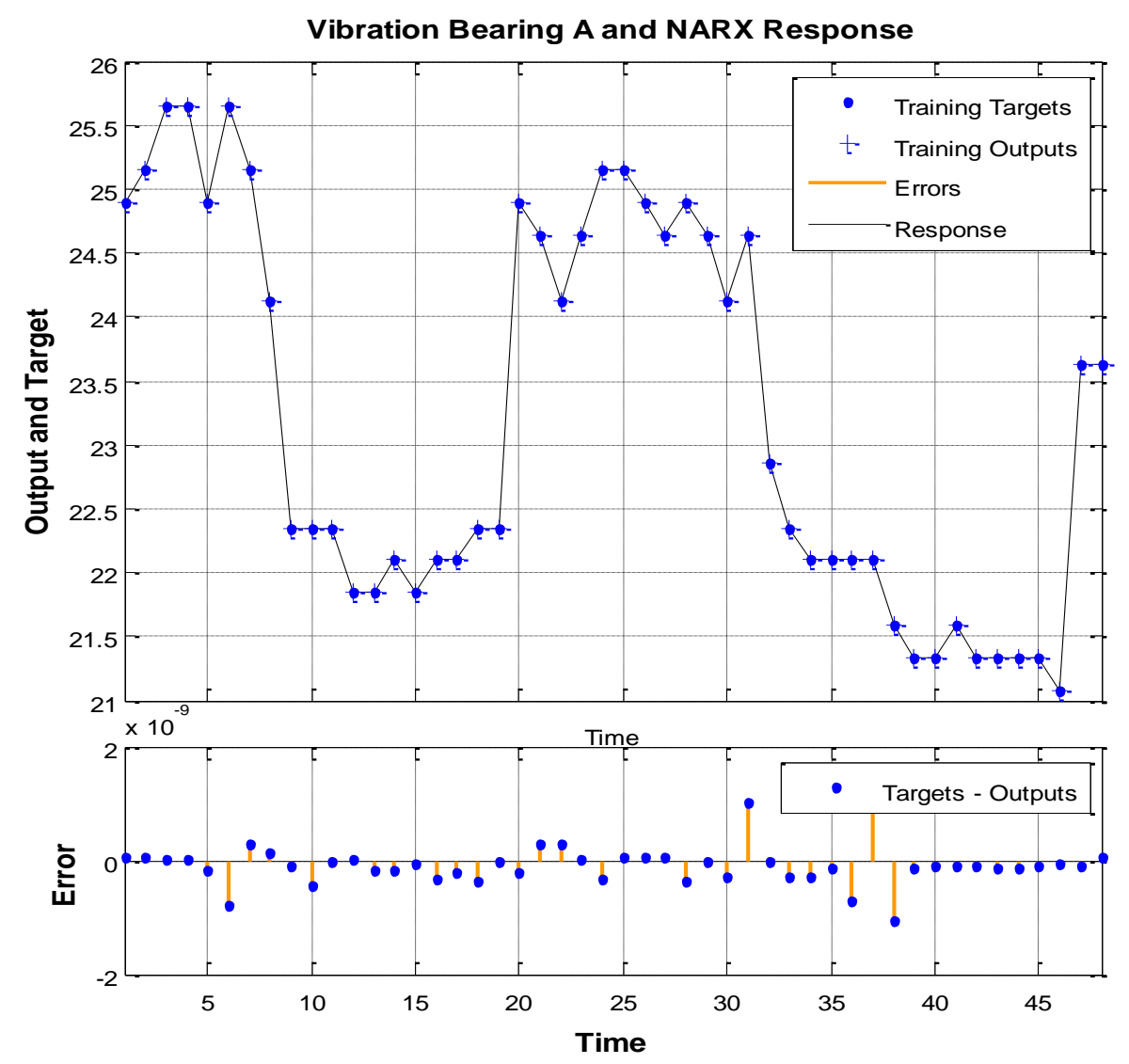

Fig. 13 Result of obtained neural model of bearing A 


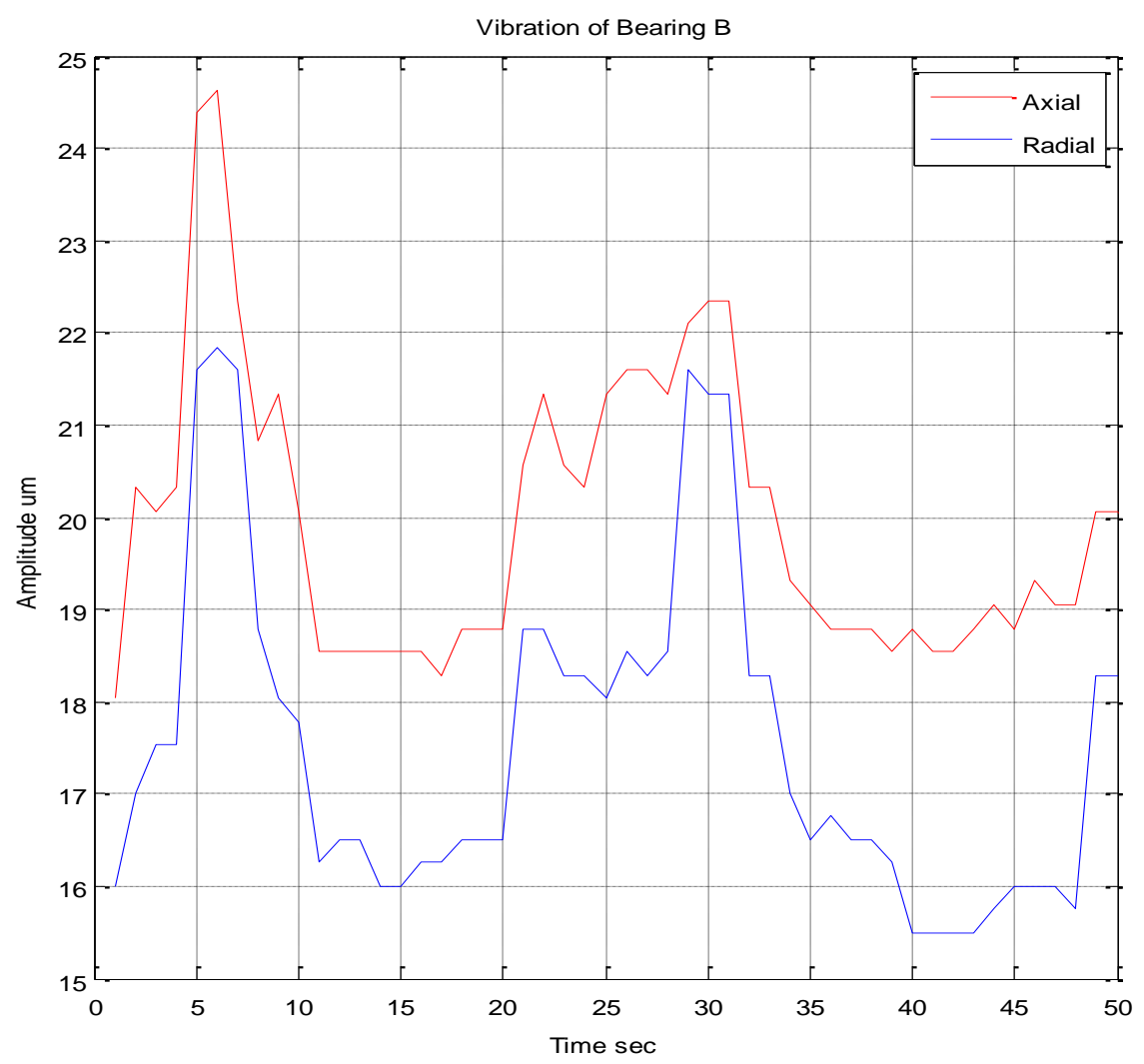

Fig. 14 Variation of axial and radial response of bearing B

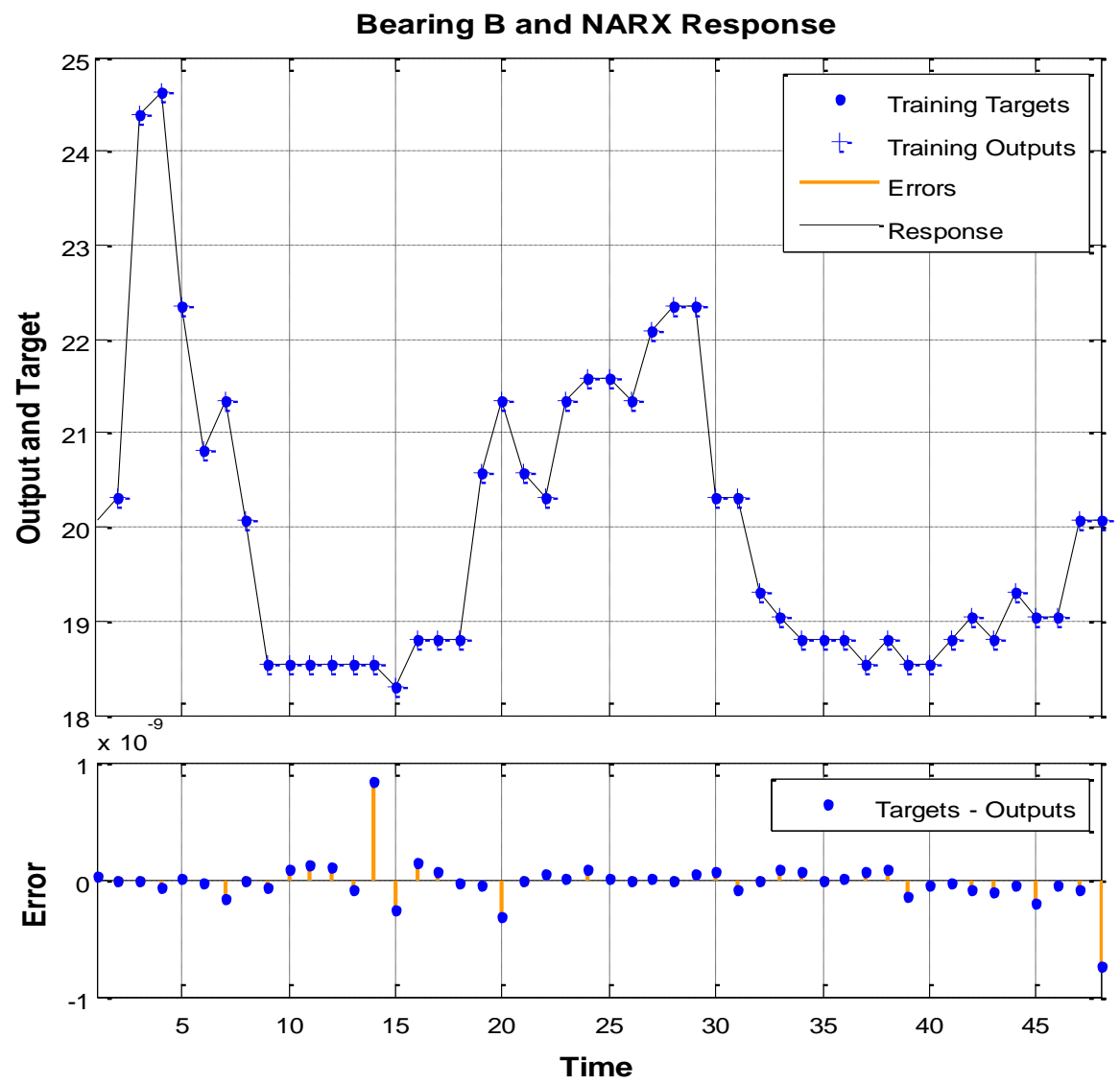

Fig. 15 Result of obtained neural model of bearing B 


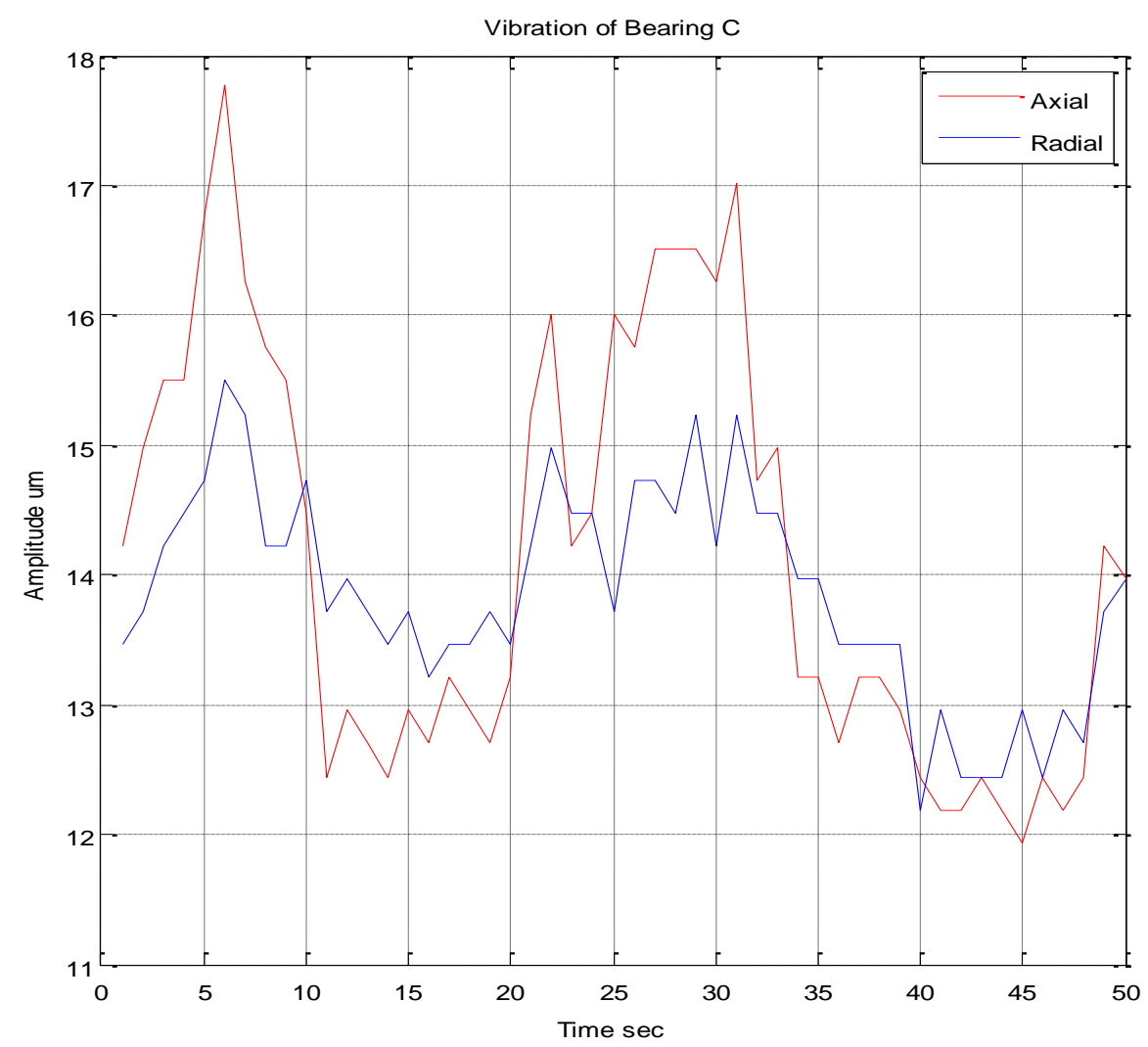

Fig. 16 Variation of axial and radial response of bearing $\mathrm{C}$

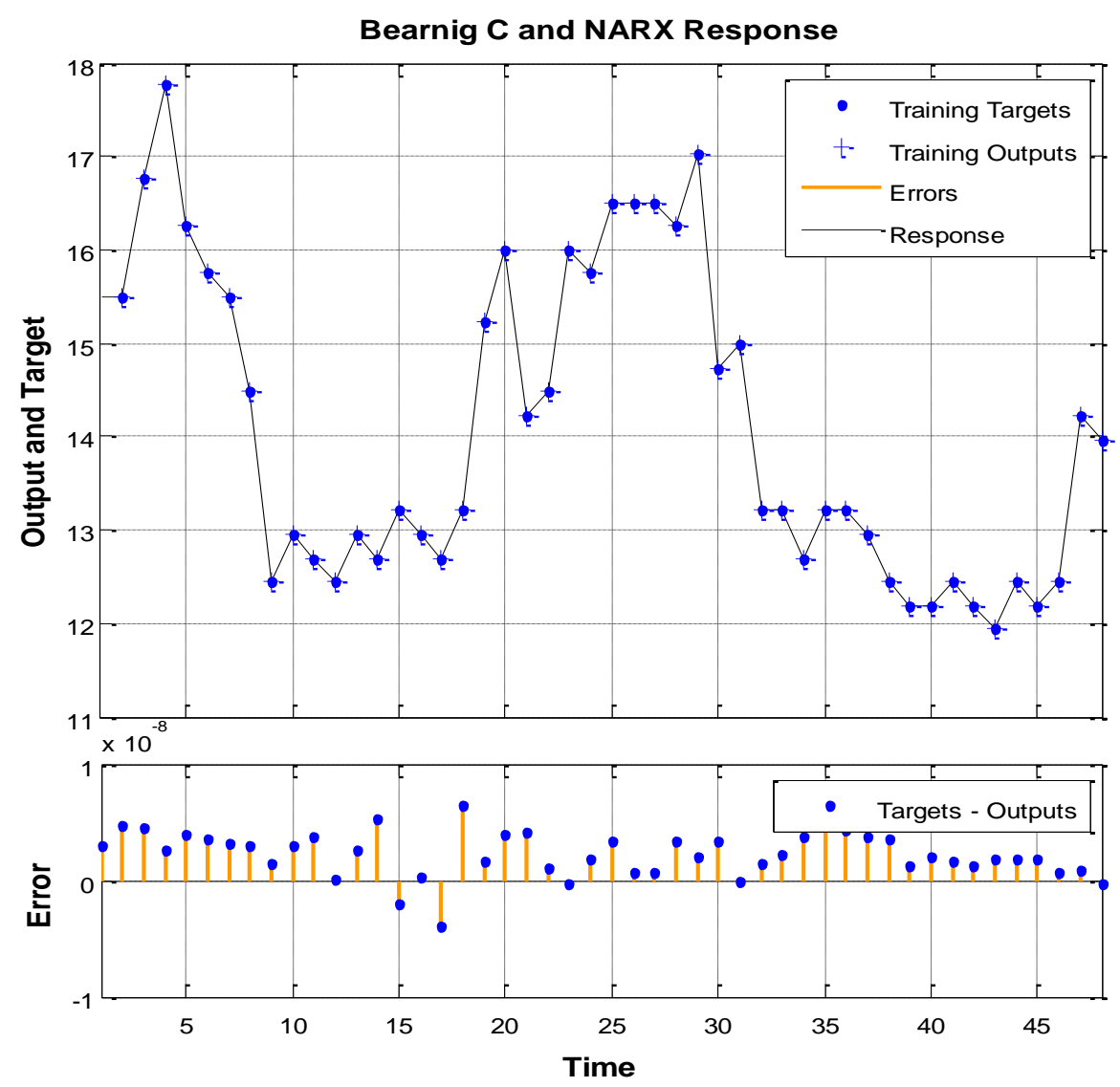

Fig. 17 Result of obtained neural model of bearing C 


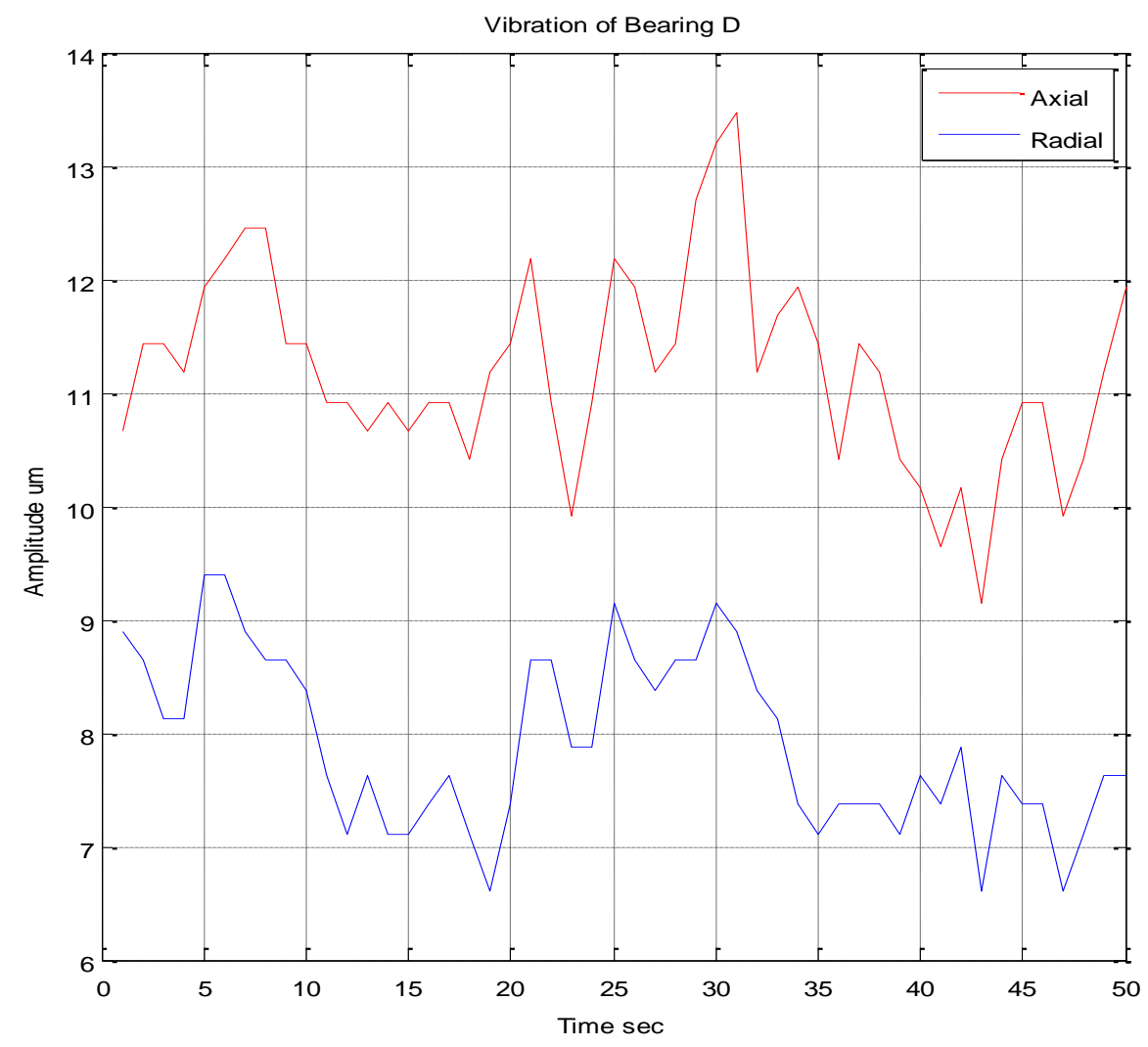

Fig. 18 Variation of axial and radial response of bearing D

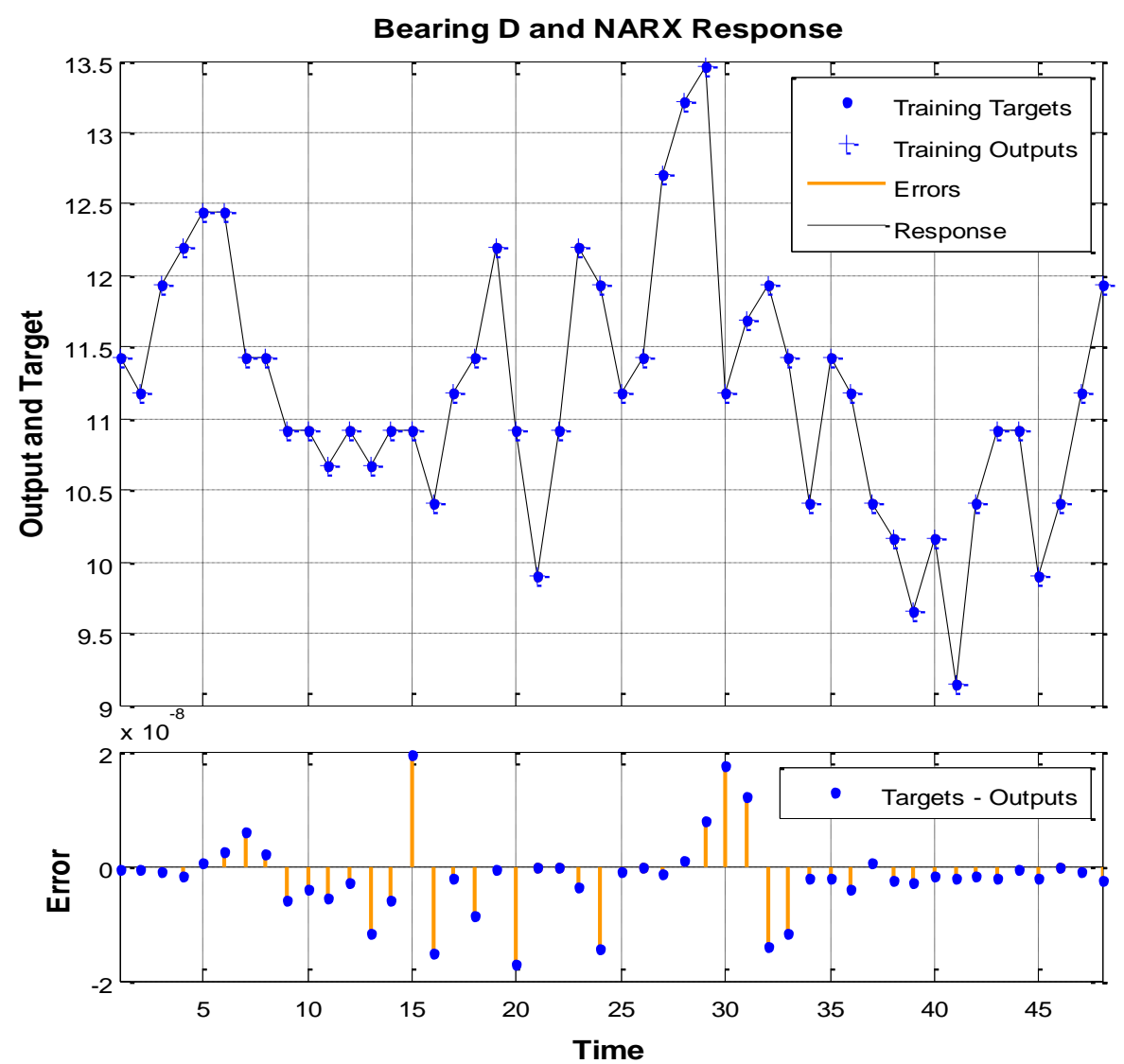

Fig. 19 Result of obtained neural model of bearing D 


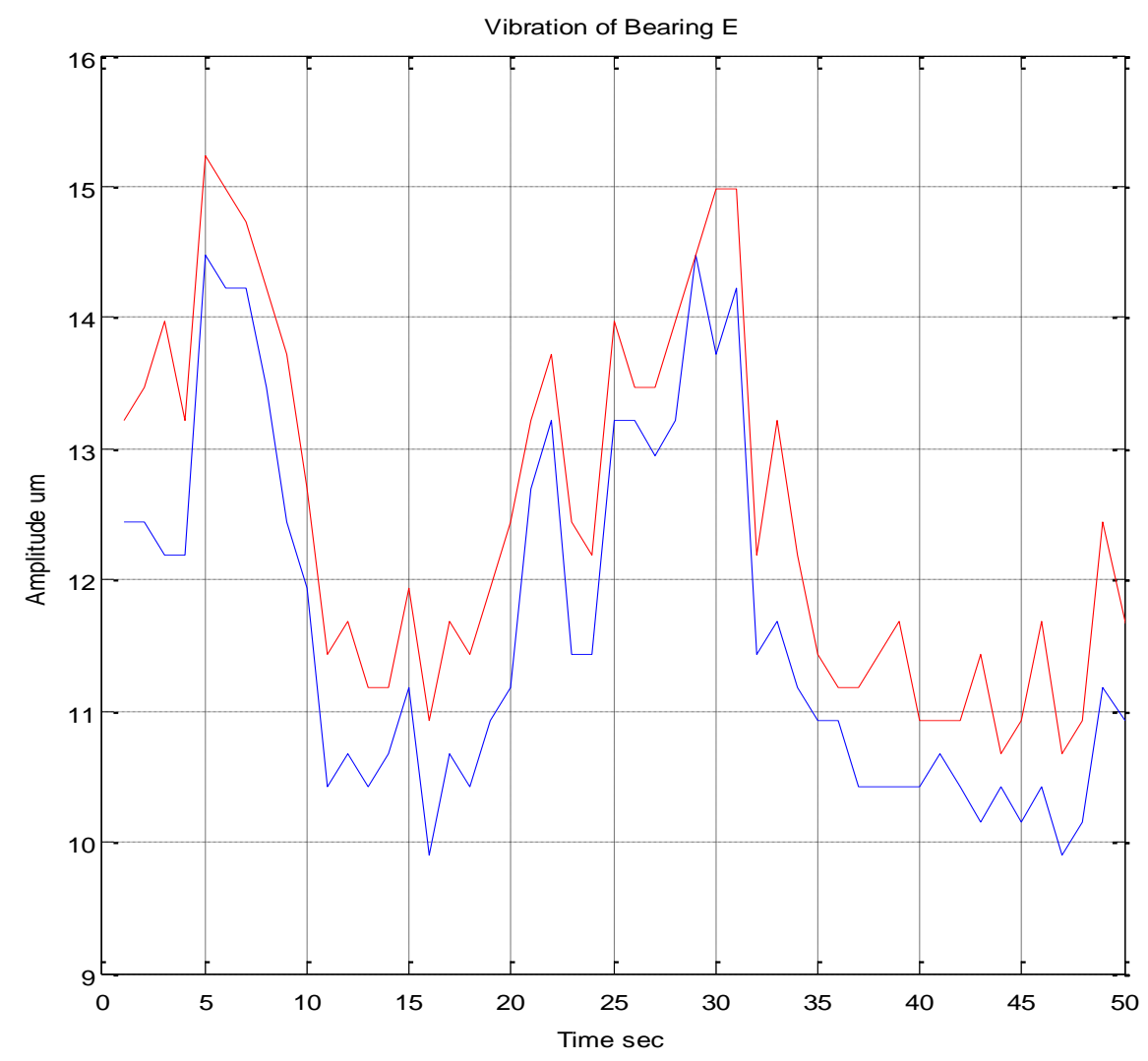

Fig. 20 Variation of axial and radial response of bearing $\mathrm{E}$

Figure 18 shows the variations of the axial and radial vibration of the bearing D responses of the low-pressure turbine with a $13.5 \mu \mathrm{m}$ axial vibration peak and Figure 19 shows the obtained neural model response of stage $\mathrm{D}$ with an acceptable modeling error, which confirms the effectiveness of the developed model for the axial and radial vibrations of bearing $\mathrm{D}$.

Figure 20 shows the variations of axial and radial vibration of the bearing $\mathrm{E}$ of the lowpressure turbine with a peak of $15.3 \mu \mathrm{m}$ of axial vibration and Figure 21 represents the obtained neural model response of bearing $\mathrm{E}$, the verification of the correct functioning of the obtained model is done using the neuronal model in loop mode and justify by the obtained modeling error, which confirms the efficiency of this model for the axial and radial vibrations of bearing $\mathrm{E}$.

In all cases of the different bearings (A, B, C, D and E) the obtained results in normal or degraded mode are very satisfactory but are of major interest for the axial or radial vibration modeling of these bearings from tested gas turbine. In a practice way and from the point of view of the neural model, we have developed an original architecture, which consists in developing a network for each stage of the turbine and then associating these five networks in a global model to realize axial and radial vibration predictions of these bearings of the turbine. Where, the model inputs are the operating conditions of this rotating machine. The performances reported in Table 2 are measured through the back propagation learning algorithm, this table shows that all the configurations tested for the different five stages of the gas turbine examined see their performances improve by using this type of learning and that the squared error results show their acceptable accuracy. 


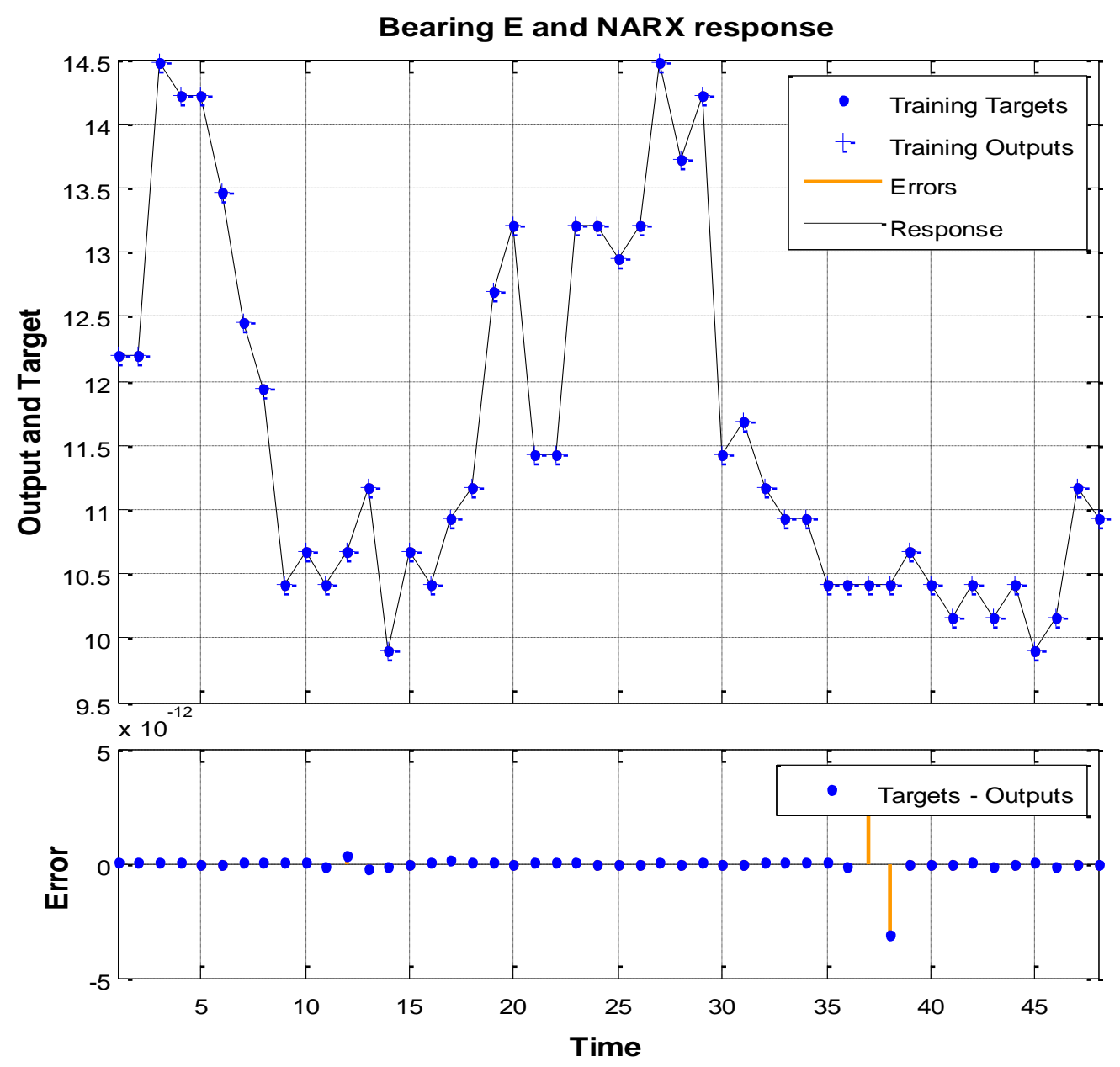

Fig. 21 Result of obtained neural model of bearing E

Table 2: Mean square error modeling

\begin{tabular}{c|c|c|c|c|c}
\hline & $\begin{array}{c}\text { Vibration } \\
\text { Bearing A }\end{array}$ & $\begin{array}{c}\text { Vibration } \\
\text { Bearing B }\end{array}$ & $\begin{array}{c}\text { Vibration } \\
\text { Bearing C }\end{array}$ & $\begin{array}{c}\text { Vibration } \\
\text { Bearing D }\end{array}$ & $\begin{array}{c}\text { Vibration } \\
\text { Bearing E }\end{array}$ \\
\hline $\begin{array}{c}\text { Mean } \\
\text { Equare } \\
\text { Error }\end{array}$ & $8.10 \mathrm{e}-28$ & $7.27 \mathrm{e}-19$ & $1.55 \mathrm{e}-20$ & $4.86 \mathrm{e}-18$ & $6.047 \mathrm{e}-29$ \\
\hline
\end{tabular}

\section{CONCLUSION}

The increasing complexity of industrial plants using gas turbines and their severe operating constraints have increased to the operation of these systems by adding values to these supervisory strategies. Moreover, this work is part of an industrial diagnostic context in which it is difficult to have knowledge about the phenomena of instability and the opposite of acquiring data on this equipment. The interest of the approach proposed in this work was to develop a diagnostic system based on dynamic neural networks, using the non-linear selfregenerating technique with high-quality exogenous input (NARX) for the modeling of the 
vibrations of a gas turbine and guarantee their profitability. This approach allows real-time diagnosis, designed to avoid failures and damage, in order to increase their availability and enable them to have good productivity, using the major interest of neural networks, which resides in its ability to analyze the failure of the turbine under examination, illustrated on real data of this turbine, which allow the synchronization of the actions of the monitoring modules of this rotating machine. And to put in place this vibration monitoring strategy, which is a challenge for the development of monitoring of the degradation state of these industrial systems, taking into account vibration phenomena and fault indicators, also have been used to provide the predictions of failure in a specific way.

\section{REFERENCES}

[1] A. Zadeh Shirazi, M. Hatami, M. Yaghoobi, S. J. S. M. Chabok. An intelligent approach to predict vibration rate in a real gas turbine. Intelligent Industrial Systems 2016 (2), No. $3,253-267$.

[2] A. Hafaifa, M. Guemana, A. Daoudi. Vibration supervision in gas turbine based on parity space approach to increasing efficiency. Journal of Vibration and Control 2015 (21), $1622-1632$.

[3] S. Madhavan, J. Rajeev, C. Sujatha, A. S. Sekhar. Vibration based damage detection of rotor blades in a gas turbine engine. Engineering Failure Analysis 2014 (46), 26 - 39.

[4] F. Z. Sierra-Espinosa, J. C. García. Vibration failure in admission pipe of a steam turbine due to flow instability. Engineering Failure Analysis 2013 (27), 30 - 40.

[5] V. S. Luk'yanov, G. A. Khodakov. Mathematical model for evaluating the natural vibration frequency of gas-turbine engine disks with small change of web thickness. Strength of Materials 1987 (19), No. 3, 430 - 434.

[6] S. Delvecchio, P. Bonfiglio, F. Pompoli. Vibro-acoustic condition monitoring of Internal Combustion Engines: A critical review of existing techniques. Mechanical Systems and Signal Processing 2018 (99), 661 - 683.

[7] Y. Wu, H. Zhang, L. Zuo. Thermoelectric energy harvesting for the gas turbine sensing and monitoring system. Energy Conversion and Management 2018 (157), 215 - 223.

[8] Z. Guo, K. Feng, T. Liu, P. Lyu, T. Zhang. Nonlinear dynamic analysis of rigid rotor supported by gas foil bearings: Effects of gas film and foil structure on subsynchronous vibrations. Mechanical Systems and Signal Processing 2018 (107), 549 - 566.

[9] L. Pesaresi, L. Salles, A. Jones, J. S. Green, C. W. Schwingshackl. Modelling the nonlinear behaviour of an underplatform damper test rig for turbine applications. Mechanical Systems and Signal Processing 2017 (85), 662 - 679.

[10] N. Hadroug, A. Hafaifa, K. Abdellah, A. Chaibet. Dynamic model linearization of two shafts gas turbine via their input / output data around the equilibrium points. Energy 2017 (120), 488 - 497.

[11] M. Tahan, E. Tsoutsanis, M. Muhammad, Z.A. Abdul Karim. Performance-based health monitoring, diagnostics and prognostics for condition-based maintenance of gas turbines: A review. Applied Energy 2017 (198), 122 - 144.

[12] M. Amozegar, K. Khorasani. An ensemble of dynamic neural network identifiers for fault detection and isolation of gas turbine engines. Neural Networks 2016 (76), 106 121. 
[13] B. Djaidir, A. Hafaifa, K. Abdallah. Faults detection in gas turbine rotor using vibration analysis under varying conditions. Journal of Theoretical and Applied Mechanics 2017 (55), No. 2, $393-406$.

[14] R. B. Joly, S. O. T. Ogaji, R. Singh, S. D. Probert. Gas-turbine diagnostics using artificial neural-networks for a high bypass ratio military turbofan engine. Applied Energy 2004 (78), No. 4, 397 - 418.

[15] P. Kin Wong, Z. Yang, Ch. M. Vong, J. Zhong. Real-time fault diagnosis for gas turbine generator systems using extreme learning machine. Neurocomputing 2014 (128), $249-257$.

[16] S. Sina Tayarani-Bathaie, K. Khorasani. Fault detection and isolation of gas turbine engines using a bank of neural networks. Journal of Process Control 2015 (36), 22-41.

[17] A. V. Kochergin, i. V. Ivshin, A. R. Sabirov, V. A. Gavrilov. Diagnostics of gas turbine engine blades using the forced vibration method. Russian Aeronautics 2007 (50), No. 3, $330-332$.

[18] M. Šofer, R. Fajkoš, R. Halama. Influence of Induction Hardening on Wear Resistance in Case of Rolling Contact. Journal of Mechanical Engineering - Strojnícky časopis 2016 (66), No. 1, 17 - 26.

[19] A. Benyounes, A. Hafaifa, M. Guemana. Gas turbine modelling based on fuzzy clustering algorithm using experimental data. Journal of Applied Artificial Intelligence 2016 (30), No. 1, $29-51$.

[20] A. K. Mohapatra, J. Sanjay Arab. Analytical investigation of parameters affecting the performance of cooled gas turbine cycle with evaporative cooling of inlet air. Arabian Journal for Science and Engineering 2013 (38), No. 6, 1587 - 1597.

[21] G. Sanjay Barad, P. V. Ramaiah, R. K. Giridhar, G. Krishnaiah. Neural network approach for a combined performance and mechanical health monitoring of a gas turbine engine. Mechanical Systems and Signal Processing 2012 (27), 729 - 742.

[22] J. Jablonská, M. Kozubková, B. Zavadilová, L. Zavadil, S. Fialová. The Investigation of the Cavitation Phenomenon in the Laval Nozzle with Full and Partial Surface Wetting. Journal of Mechanical Engineering - Strojnicky časopis 2017 (67) 67, No. 1, 55 - 68.

[23] Md Sazzad Hossain, Zhi Chao Ong, Zubaidah Ismail, Siamak Noroozi, Shin Yee Khoo. Artificial neural networks for vibration based inverse parametric identifications: A review. Applied Soft Computing 2017 (52), 203 - 219.

[24] Fethi Bellamine, A. Almansoori, A. Elkamel. Modeling of complex dynamic systems using differential neural networks with the incorporation of a priori knowledge. Applied Mathematics and Computation 2015 (266), 515 - 526.

[25] E. Swiercz. Classification of parameter changes in a dynamic system with the use of wavelet analysis and neural networks. Advances in Engineering Software 2012 (45), No. $1,28-41$.

[26] H. Nikpey, M. Assadi, P. Breuhaus. Development of an optimized artificial neural network model for combined heat and power micro gas turbines. Applied Energy 2013 (108), $137-148$.

[27] M. Pástor, P. Frankovský, M. Hagara, P. Lengvarský. The use of optical methods in the analysis of the areas with stress concentration. Journal of Mechanical Engineering Strojnícky časopis 2018 (68), No. 2, 61 - 76. 
[28] H. Asgari, X. Chen, M. Morini, M. Pinelli, R. Sainudiin, P. Ruggero Spina, M. Venturini. NARX models for simulation of the start-up operation of a single-shaft gas turbine. Applied Thermal Engineering 2016 (93), 368 - 376.

[29] Z. N. Sadough Vanini, K. Khorasani, N. Meskin. Fault detection and isolation of a dual spool gas turbine engine using dynamic neural networks and multiple model approach. Information Sciences 2014 (259), 234 - 251. 